\title{
Synthesis of 5-Amino-2,5-dihydro-1H-benzo[b]azepines Using a One- Pot Multibond Forming Process
}

\author{
Salaheddin A. I. Sharif, Ewen D. D. Calder, Fábio G. Delolo, and Andrew Sutherland* \\ WestCHEM, School of Chemistry, The Joseph Black Building, University of Glasgow, Glasgow G12 8QQ, United Kingdom
}

Supporting Information

ABSTRACT: Rapid access to allylic trichloroacetimidates bearing a 2-allylaminoaryl group from readily available 2iodoanilines combined with a one-pot multibond forming process has allowed the efficient synthesis of a series of 5amino-2,5-dihydro- $1 H$-benzo $[b]$ azepines. The potential of these compounds as synthetic building blocks was demonstrated by the preparation of a late-stage intermediate of the hyponatremia agent, mozavaptan.

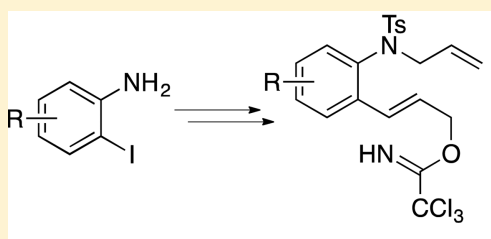
$\stackrel{\text { one-pot }}{\text { [3,3] }}$<smiles>O=C(NC1C=CC[NH2+]c2ccccc21)C(Cl)(Cl)Cl</smiles>

$(49-92 \%)$

\section{INTRODUCTION}

$1 H$-Benzo[b]azepines are an important class of sevenmembered heterocyclic compound found as a key structural element in a wide variety of pharmaceutically active substances. $^{1,2}$ Within this class, 5-amino-2,3,4,5-tetrahydro$1 \mathrm{H}$-benzo[b]azepines are of particular significance and include compounds such as mozavaptan (1), a nonpeptide vasopressin V2-receptor antagonist used for the treatment of hyponatremia (low blood sodium levels), ${ }^{3}$ and 3,5-bis(trifluoromethyl)benzyl protected 2,3,4,5-tetrahydro- $1 \mathrm{H}$-benzo[ $b]$ azepine 2 , developed for the treatment of dyslipidemia (Figure 1). ${ }^{4}$ The interest in 5-

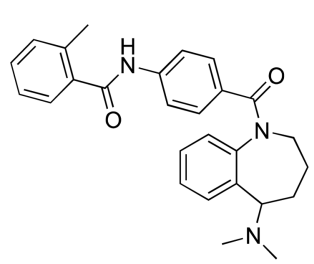

Mozavaptan (1)

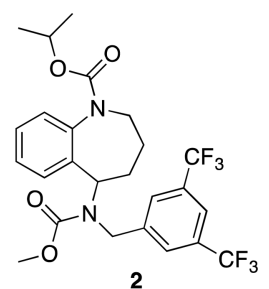

Figure 1. Structures of pharmacologically active 5-amino-2,3,4,5tetrahydro- $1 H$-benzo[b]azepines.

amino-2,3,4,5-tetrahydro- $1 H$-benzo $[b]$ azepines has led recently to a detailed analysis of their conformational bias and a greater understanding of their physicochemical properties. ${ }^{5}$

Due to the pharmacological importance of 5-amino-2,3,4,5tetrahydro- $1 H$-benzo $[b]$ azepines, a number of methods have been developed for their synthesis. ${ }^{2,3 c, 6}$ Traditionally, a Dieckmann condensation has been used to prepare $1 \mathrm{H}$ benzo $[b]$ azepin-5-ones, followed by introduction of the amino substituent by reductive amination of the ketone (Scheme 1a). ${ }^{2 \mathrm{c}}$ More recently, the azepine ring system in these compounds has been prepared using methods such as the Beckmann rearrangement, ${ }^{6 b}$ the Mitsunobu reaction, ${ }^{6 a}$ reductive ring opening of aza-bridged azepines, ${ }^{6 e}$ and ring closing metathesis (RCM) (Scheme 1b). ${ }^{6 \mathrm{~d}, 7}$ With the aim of
Scheme 1. Synthetic Approaches for the Preparation of 5Amino-Substituted $1 H$-Benzo $[b]$ azepines

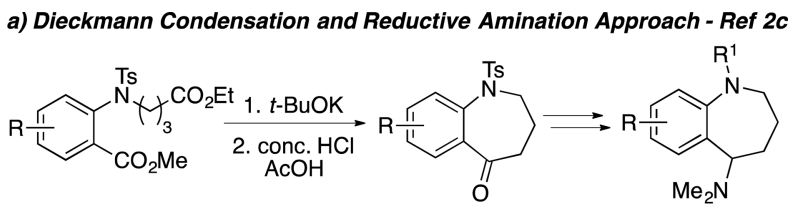

b) Stepwise Vinylation of an Imine and RCM Approach - Ref $7 \mathrm{~b}$

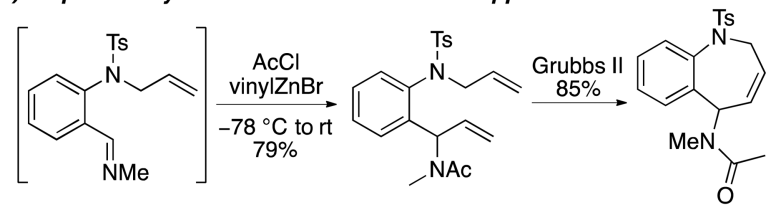

c) One-Pot Synthesis - This Work

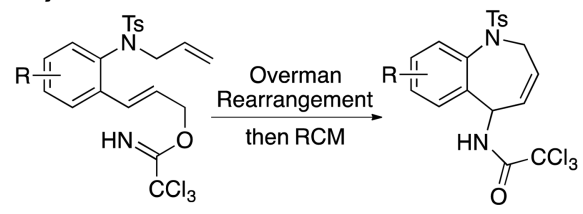

developing new methods for the preparation of highly functional polycyclic compounds, we have demonstrated that benzannulated alkene derived allylic alcohols could be used in one-pot multireaction processes for the efficient synthesis of amino-substituted indenes, dihydronaphthalenes, and 1-benzoxepines. ${ }^{8}$ We now report a short and general synthesis of allylic trichloroacetimidates bearing a 2-allylaminoaryl group from readily available 2-iodoanilines and demonstrate the application of these compounds in a one-pot multibond forming process for the efficient synthesis of 5-amino-2,5-dihydro-1H-benzo$[b]$ azepines (Scheme 1c).

Received: June 4, 2016

Published: July 14, 2016 


\section{RESULTS AND DISCUSSION}

The substrates for the one-pot process, (E)-(2-allylamino)cinnamyl alcohols, were prepared using a four-step route from commercially available 2-iodoanilines (Scheme 2). Mizoroki-

\section{Scheme 2. Synthesis of Allylic Alcohols $6 a-f^{a}$}

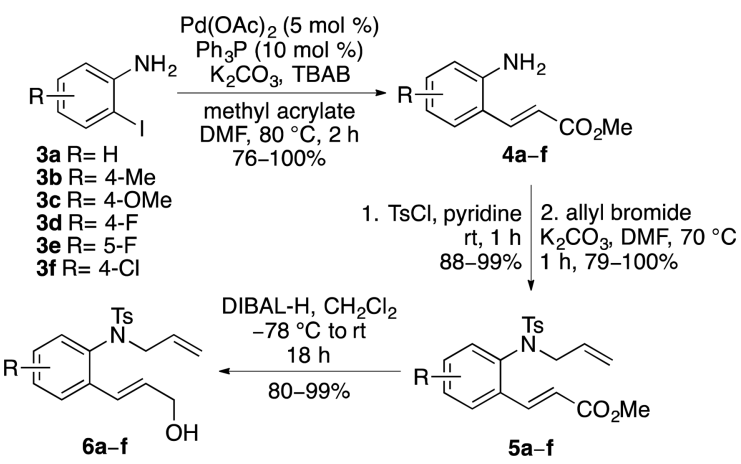

${ }^{a}$ Isolated yields are shown.

Heck reaction of 2-iodoanilines $\mathbf{3} \mathbf{a}-\mathbf{f}$ with methyl acrylate and palladium(II) acetate (5 mol \%) under standard conditions gave the corresponding methyl $(E)-2^{\prime}$-aminocinnamates $4 \mathbf{a}-\mathbf{f}$ in excellent yields $(76-100 \%))^{9,10}$ The amines were protected with the tosylate group, and this allowed monoallylation using allyl bromide and potassium carbonate. ${ }^{11}$ Finally, reduction of the (E)- $\alpha, \beta$-unsaturated methyl esters $5 \mathbf{a}-\mathbf{f}$ with DIBAL-H gave (E)-(2-allylamino)cinnamyl alcohols $\mathbf{6 a}-\mathbf{f}$ in high overall yields.

While this synthetic route allowed access to a range of $(E)$ (2-allylamino)cinnamyl alcohols, the preparation of a $4^{\prime}$-nitro analogue was not possible. Attempted Mizoroki-Heck coupling of 2-iodo-4-nitroaniline with methyl acrylate instead gave the conjugate addition product. An alternative approach was developed for this compound (Scheme 3). 2-Chloro-5-

Scheme 3. Synthesis of Allylic Alcohol $6 \mathrm{~g}^{a}$

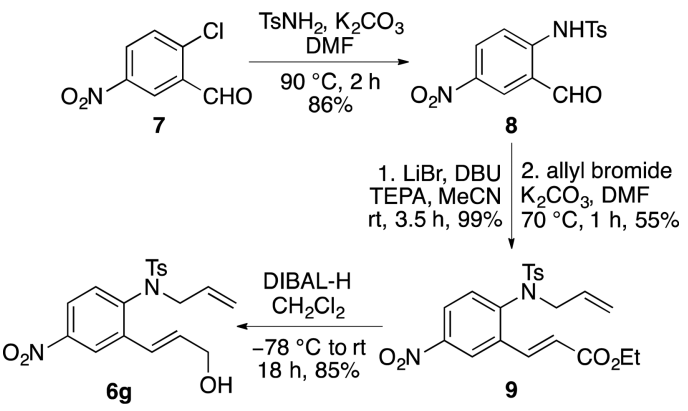

${ }^{a}$ Isolated yields are shown.

nitrobenzaldehyde (7) was subjected to a nucleophilic aromatic substitution reaction with $p$-toluenesulfonamide, which gave 8 in $86 \%$ yield. $^{12}$ Horner-Wadsworth-Emmons reaction of 8 under Masamune-Roush conditions with triethyl phosphonoacetate (TEPA) gave the ethyl (E)-2'-aminocinnamate in quantitative yield. ${ }^{13}$ Analysis of the ${ }^{1} \mathrm{H}$ NMR spectrum of the crude reaction mixture showed exclusive formation of the $E$ alkene. Allylation of the amino group was then performed under the same conditions as before. However, due to decreased nucleophilicity of this compound, the product was isolated in a modest $55 \%$ yield. DIBAL-H reduction of the ethyl ester then completed the four-step synthesis of nitrosubstituted cinnamyl alcohol $\mathbf{6 g}$.

Having prepared a small library of (E)-(2-allylamino)cinnamyl alcohols, 6a was used for optimization of the onepot process (Table 1). Based on previous work, ${ }^{8,14}$ the

Table 1. Optimization of the One-Pot Process ${ }^{a}$

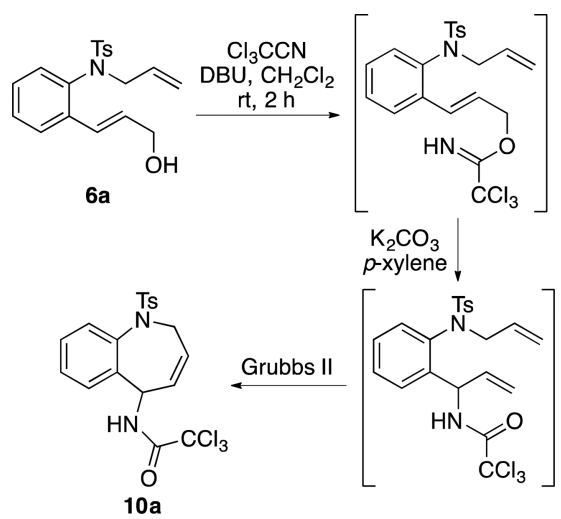

$\begin{array}{cccc}\begin{array}{c}\text { Overman } \\ \text { entry }\end{array} & \begin{array}{c}\text { rearrangement } \\ 1\end{array} & \text { RCM reaction } & \begin{array}{c}\text { yield } \\ (\%)^{a}\end{array} \\ 2 & 140{ }^{\circ} \mathrm{C}, 48 \mathrm{~h} & \text { Grubbs II }\left(\begin{array}{c}10 \mathrm{~mol} \mathrm{\%}), 50{ }^{\circ} \mathrm{C}, \\ 48 \mathrm{~h}\end{array}\right. & 69 \\ 3 & 160{ }^{\circ} \mathrm{C}, 24 \mathrm{~h} & \text { Grubbs II }\left(\begin{array}{c}10 \mathrm{~mol} \%), 50{ }^{\circ} \mathrm{C}, \\ 48 \mathrm{~h}\end{array}\right. & 70 \\ 4 & 160{ }^{\circ} \mathrm{C}, 24 \mathrm{~h} & \text { Grubbs II }(2.5 \mathrm{~mol} \mathrm{\%}), 60{ }^{\circ} \mathrm{C}, & 58 \\ 48 \mathrm{~h} & \text { Grubbs II }(5 \mathrm{~mol} \%), 60{ }^{\circ} \mathrm{C}, 18 \mathrm{~h} & 81 \\ { }^{a} \text { Isolated yields are shown. } & & \end{array}$

thermally mediated Overman rearrangement was performed at $140{ }^{\circ} \mathrm{C}$ and the RCM step was done using Grubbs' second generation catalyst $(10 \mathrm{~mol} \%)$ (entry 1$).{ }^{15}$ While this gave a yield of $69 \%$ over the three steps, both the rearrangement and metathesis stages required reaction times of $48 \mathrm{~h}$. Increasing the temperature of the Overman rearrangement to $160{ }^{\circ} \mathrm{C}$ allowed a shorter reaction time $(24 \mathrm{~h})$ with a similar overall yield (entry 2 ). The catalyst loading and temperature of the RCM step was then investigated. It was found that a catalyst loading of $5 \mathrm{~mol}$ $\%$ and a temperature of $60{ }^{\circ} \mathrm{C}$ was optimal for the RCM step, with the reaction complete after $18 \mathrm{~h}$ (entry 4). Using the optimized conditions for both key steps gave 5-amino-2,5dihydro- $1 H$-benzo[ $b]$ azepine 10 a in $81 \%$ yield from $\mathbf{6 a}$.

Using the optimized one-pot procedure, the scope of the process with various (E)-(2-allylamino) cinnamyl alcohol substrates was explored (Scheme 4). Overall, the process was found to be general and high yielding (79-92\%) for the preparation of 5-amino-2,5-dihydro-1H-benzo[ $b]$ azepines bearing a range of substituents. Only in the case of the strongly electron-deficient $4^{\prime}$-nitrophenyl analogue $6 \mathrm{~g}$ did the conditions require significant modification. For this compound, both key steps entailed longer reaction times and this likely accounts for the lower overall yield of $49 \%$.

The synthetic potential of these products was demonstrated with the three-step conversion of 10a to 5-amino-2,3,4,5tetrahydro- $1 H$-benzo $[b]$ azepine 12 , a late-stage intermediate for the preparation of mozavaptan and its analogues (Scheme 5). ${ }^{3}$ A one-pot procedure was used to remove the trichloroacyl group and reprotect the amine as the Boc-derivative. Hydrogenation at atmospheric pressure, followed by detosylation with magnesium under mild conditions, gave 5-amino-2,3,4,5- 
Scheme 4. Synthesis of 5-Amino-2,5-Dihydro-1Hbenzo $[b]$ azepines $10 \mathrm{~b}-\mathrm{g}^{a}$

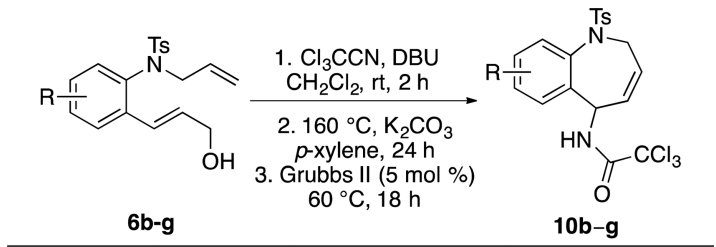<smiles>Cc1ccc2c(c1)C(NC(=O)C(Cl)(Cl)Cl)C=CCN2C</smiles><smiles>COc1ccc2c(c1)C(NC(=O)Cl)C=CCN2C</smiles><smiles>CN1CC=CC(NC(=O)C(Cl)(Cl)Cl)c2cc(F)ccc21</smiles><smiles>[13CH3]N1CC=CC(NC(=O)C(Cl)(Cl)Cl)c2ccc(F)cc21</smiles>

$10 \mathrm{e}, 92 \%$<smiles>CC(C)(C)O[As](=O)([O-])N1CC=CC(NC(=O)C(Cl)(Cl)Cl)c2cc(Cl)ccc21</smiles><smiles>[13CH3]N1CC=CC(NC(=O)C(Cl)(Cl)Cl)c2cc([N+](=O)[O-])ccc21</smiles>

10g, $49 \% c$

${ }^{a}$ Isolated yields are shown. ${ }^{b}$ The RCM step required a reaction time of $24 \mathrm{~h} .{ }^{c}$ The Overman rearrangement and RCM step required reaction times of 43 and $31 \mathrm{~h}$, respectively.

Scheme 5. Formal Synthesis of Mozavaptan $(1)^{a}$

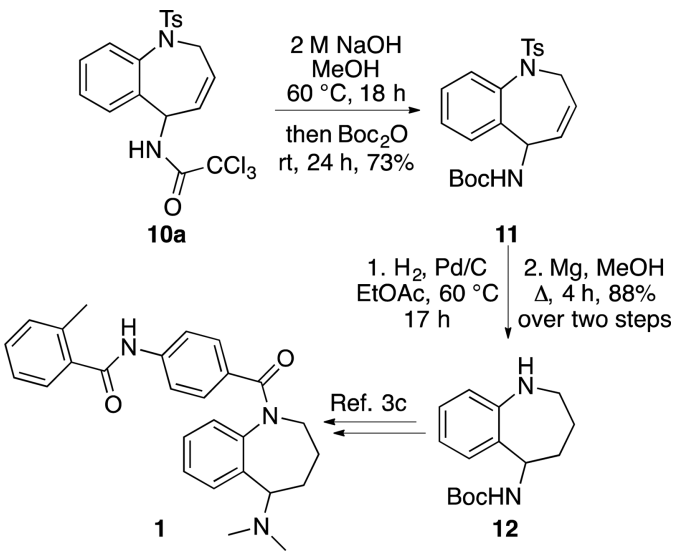

${ }^{a}$ Isolated yields are shown.

tetrahydro- $1 H$-benzo $[b]$ azepine $\mathbf{1 2}$ in $88 \%$ yield. Overall, the highly efficient four-step route to allylic alcohol $\mathbf{6 a}$, combined with the one-pot multibond forming strategy has allowed the synthesis of 5-amino-2,3,4,5-tetrahydro- $1 \mathrm{H}$-benzo[ $b]$ azepine $\mathbf{1 2}$ in $46 \%$ overall yield from commercially available 2 -iodoaniline (3a). Mozavaptan is easily prepared from $\mathbf{1 2}$ by benzoylation of the $1 H$-benzo[b]azepine ring nitrogen, removal of the Bocprotecting group, and reductive amination of the resulting amine with formaldehyde. ${ }^{3 c}$

\section{CONCLUSIONS}

In summary, a four-step synthesis of (E)-(2-allylamino)cinnamyl alcohols has been developed from readily available 2-iodoanilines using a highly efficient Mizoroki-Heck coupling. Following transformation to the corresponding allylic trichloroacetimidates, these compounds were converted to a series of 5amino-2,5-dihydro- $1 H$-benzo $[b]$ azepines using a one-pot multibond forming process. As demonstrated with the straightfor-

ward synthesis of 5-amino-2,3,4,5-tetrahydro- $1 H$-benzo[b]azepine 12, a late-stage intermediate for the synthesis of mozavaptan, these compounds have potential for synthetic and medicinal chemistry applications. Work is currently underway to investigate further synthetic applications of 5-amino-2,5dihydro- $1 H$-benzo[ $b]$ azepines and extend the use of one-pot multibond forming reaction processes.

\section{EXPERIMENTAL SECTION}

All reagents and starting materials were obtained from commercial sources and used as received. All dry solvents were purified using a solvent purification system. All reactions were performed under an atmosphere of argon unless otherwise mentioned. Brine refers to a saturated solution of sodium chloride. Flash column chromatography was performed using silica gel $60(35-70 \mu \mathrm{m})$. Aluminum-backed plates precoated with silica gel $60 \mathrm{~F}_{254}$ were used for thin layer chromatography and were visualized with a UV lamp or by staining with potassium permanganate. ${ }^{1} \mathrm{H}$ NMR spectra were recorded on an NMR spectrometer at either 400 or $500 \mathrm{MHz}$, and data are reported as follows: chemical shift in $\mathrm{ppm}$ relative to tetramethylsilane or the solvent $\left(\mathrm{CDCl}_{3}, \delta 7.26 \mathrm{ppm}\right)$ as the internal standard, multiplicity ( $\mathrm{s}=$ singlet, $\mathrm{d}=$ doublet, $\mathrm{t}=$ triplet, $\mathrm{q}=$ quartet, $\mathrm{m}=$ multiplet or overlap of nonequivalent resonances, integration). ${ }^{13} \mathrm{C}$ NMR spectra were recorded on an NMR spectrometer at either 101 or $126 \mathrm{MHz}$, and data are reported as follows: chemical shift in ppm relative to tetramethylsilane or the solvent $\left(\mathrm{CDCl}_{3}, \delta 77.0 \mathrm{ppm}\right)$ as the internal standard, multiplicity with respect to hydrogen (deduced from DEPT experiments, $\mathrm{C}, \mathrm{CH}, \mathrm{CH}_{2}$, or $\mathrm{CH}_{3}$ ). Infrared spectra were recorded on an FTIR spectrometer; wavenumbers are indicated in $\mathrm{cm}^{-1}$. Mass spectra were recorded using the electrospray technique. HRMS spectra were recorded using a dual-focusing magnetic analyzer mass spectrometer. Melting points are uncorrected.

Methyl (2E)-3-(2'-Aminophenyl)prop-2-enoate (4a). ${ }^{10}$ Methyl acrylate $(1.53 \mathrm{~mL}, 18.3 \mathrm{mmol})$ was added to a solution of 2iodoaniline (3a) $(2.00 \mathrm{~g}, 9.13 \mathrm{mmol})$, palladium acetate $(0.110 \mathrm{~g}$, $0.460 \mathrm{mmol})$, triphenylphosphine $(0.239 \mathrm{~g}, 0.913 \mathrm{mmol})$, potassium carbonate $(1.26 \mathrm{~g}, 9.13 \mathrm{mmol})$, and tetrabutylammonium bromide $(0.741 \mathrm{~g}, 2.30 \mathrm{mmol})$ in $N, N^{\prime}$-dimethylformamide $(90 \mathrm{~mL})$. The reaction mixture was stirred at $80{ }^{\circ} \mathrm{C}$ for $2 \mathrm{~h}$. The mixture was cooled to room temperature, diluted with water $(50 \mathrm{~mL})$, and extracted with diethyl ether $(3 \times 50 \mathrm{~mL})$. The organic layer was washed with $5 \%$ aqueous lithium chloride solution $(10 \mathrm{~mL})$ and brine $(10 \mathrm{~mL})$, dried $\left(\mathrm{MgSO}_{4}\right)$, filtered, and concentrated in vacuo. The residue was purified by column chromatography (diethyl ether/petroleum ether, $1: 4)$ to give methyl (2E)-3-(2'-aminophenyl)prop-2-enoate (4a) (1.59 g, $99 \%$ ) as a yellow solid. Mp $64-66{ }^{\circ} \mathrm{C} ; R_{f}=0.33$ (diethyl ether) petroleum ether $=1: 1)$. Spectroscopic data were consistent with the literature. ${ }^{10}{ }^{1} \mathrm{H}$ NMR $\left(400 \mathrm{MHz}, \mathrm{CDCl}_{3}\right) \delta 3.80(\mathrm{~s}, 3 \mathrm{H}), 3.98$ (br s, $2 \mathrm{H}), 6.36(\mathrm{~d}, J=15.8 \mathrm{~Hz}, 1 \mathrm{H}), 6.70(\mathrm{dd}, J=8.0,1.3 \mathrm{~Hz}, 1 \mathrm{H}), 6.77$ (ddd, $J=8.0,7.3,1.3 \mathrm{~Hz}, 1 \mathrm{H}), 7.17(\mathrm{ddd}, J=7.9,7.3,1.3 \mathrm{~Hz}, 1 \mathrm{H})$, $7.38(\mathrm{dd}, J=7.9,1.3 \mathrm{~Hz}, 1 \mathrm{H}), 7.83(\mathrm{~d}, J=15.8 \mathrm{~Hz}, 1 \mathrm{H}) ;{ }^{13} \mathrm{C}$ NMR $\left(101 \mathrm{MHz}, \mathrm{CDCl}_{3}\right) \delta 51.7\left(\mathrm{CH}_{3}\right), 116.7(\mathrm{CH}), 117.7(\mathrm{CH}), 119.0$ (CH), $119.9(\mathrm{C}), 128.1(\mathrm{CH}), 131.3(\mathrm{CH}), 140.3(\mathrm{CH}), 145.6(\mathrm{C})$, 167.7 (C); MS (ESI) $m / z 200\left(\mathrm{MNa}^{+}, 4\right), 168$ (26), 146 (100), 128 (31).

Methyl (2E)-3-(2'-Amino-5'-methylphenyl)prop-2-enoate (4b). ${ }^{16}$ The reaction was carried out as described for the synthesis of methyl (2E)-3-(2'-aminophenyl)prop-2-enoate (4a) using 4methyl-2-iodoaniline $(3 \mathbf{b})(2.00 \mathrm{~g}, 8.58 \mathrm{mmol})$. Purification by column chromatography (diethyl ether/petroleum ether, 1:3) gave methyl (2E)-3-(2'-amino-5'-methylphenyl)prop-2-enoate (4b) (1.64 g, $100 \%$ ) as a yellow solid. $\mathrm{Mp} 84-86{ }^{\circ} \mathrm{C} ; R_{f}=0.28$ (diethyl ether/ petroleum ether $=1: 1)$. Spectroscopic data were consistent with the literature. ${ }^{16}{ }^{1} \mathrm{H} \mathrm{NMR}\left(400 \mathrm{MHz}, \mathrm{CDCl}_{3}\right) \delta 2.24(\mathrm{~s}, 3 \mathrm{H}), 3.79(\mathrm{~s}, 3 \mathrm{H})$, $3.86($ br s, $2 \mathrm{H}), 6.34(\mathrm{~d}, J=15.8 \mathrm{~Hz}, 1 \mathrm{H}), 6.62(\mathrm{~d}, J=8.1 \mathrm{~Hz}, 1 \mathrm{H})$, $6.99(\mathrm{dd}, J=8.1,1.5 \mathrm{~Hz}, 1 \mathrm{H}), 7.19(\mathrm{~d}, J=1.5 \mathrm{~Hz}, 1 \mathrm{H}), 7.82(\mathrm{~d}, J=$ $15.8 \mathrm{~Hz}, 1 \mathrm{H}) ;{ }^{13} \mathrm{C}$ NMR $\left(101 \mathrm{MHz}, \mathrm{CDCl}_{3}\right) \delta 20.4\left(\mathrm{CH}_{3}\right), 51.6$ $\left(\mathrm{CH}_{3}\right), 117.0(\mathrm{CH}), 117.4(\mathrm{CH}), 119.9(\mathrm{C}), 128.2(\mathrm{C}), 128.2(\mathrm{CH})$, 
$132.3(\mathrm{CH}), 140.4(\mathrm{CH}), 143.3$ (C), 167.8 (C); MS (ESI) $m / z 214$ $\left(\mathrm{MNa}^{+}, 100\right), 192$ (11), $182(23)$.

Methyl (2E)-3-(2'-Amino-5'-methoxyphenyl)prop-2-enoate (4c). ${ }^{17}$ The reaction was carried out as described for the synthesis of methyl (2E)-3-(2'-aminophenyl)prop-2-enoate (4a) using 4methoxy-2-iodoaniline $(3 \mathrm{c})(0.170 \mathrm{~g}, 0.680 \mathrm{mmol})$ and potassium carbonate $(0.188 \mathrm{~g}, 1.36 \mathrm{mmol})$. Purification by column chromatography (diethyl ether/petroleum ether, 1:3) gave methyl (2E)-3-(2'amino-5'-methoxyphenyl)prop-2-enoate (4c) $(0.141 \mathrm{~g}, 100 \%)$ as a yellow solid. Mp $93-95^{\circ} \mathrm{C}$; $R_{f}=0.20$ (diethyl ether/petroleum ether $=1: 1)$. Spectroscopic data were consistent with the literature. ${ }^{17}{ }^{1} \mathrm{H}$ NMR (400 MHz, $\left.\mathrm{CDCl}_{3}\right) \delta 3.71($ br s, $2 \mathrm{H}), 3.76(\mathrm{~s}, 3 \mathrm{H}), 3.81(\mathrm{~s}$, $3 \mathrm{H}), 6.35(\mathrm{~d}, J=15.8 \mathrm{~Hz}, 1 \mathrm{H}), 6.67(\mathrm{~d}, J=8.7 \mathrm{~Hz}, 1 \mathrm{H}), 6.82(\mathrm{dd}, J=$ 8.7, $2.9 \mathrm{~Hz}, 1 \mathrm{H}), 6.92(\mathrm{~d}, J=2.9 \mathrm{~Hz}, 1 \mathrm{H}), 7.82(\mathrm{~d}, J=15.8 \mathrm{~Hz}, 1 \mathrm{H})$; ${ }^{13} \mathrm{C}$ NMR $\left(101 \mathrm{MHz}, \mathrm{CDCl}_{3}\right) \delta 51.7\left(\mathrm{CH}_{3}\right), 55.8\left(\mathrm{CH}_{3}\right), 111.6(\mathrm{CH})$, $117.9(\mathrm{CH}), 118.4(\mathrm{CH}), 118.7(\mathrm{CH}), 120.8(\mathrm{C}), 139.6(\mathrm{C}), 140.2$ (CH), 152.8 (C), $167.6(\mathrm{C})$; MS (ESI) $m / z 208\left(\mathrm{MH}^{+}, 100\right)$.

Methyl (2E)-3-(2'-Amino-5'-fluorophenyl)prop-2-enoate (4d). ${ }^{10}$ The reaction was carried out as described for the synthesis of methyl (2E)-3-(2'-aminophenyl)prop-2-enoate (4a) using 4-fluoro2-iodoaniline (3d) $(3.77 \mathrm{~g}, 16.0 \mathrm{mmol})$ and potassium carbonate $(4.40$ g, $32.0 \mathrm{mmol}$ ). Purification by column chromatography (ethyl acetate/ petroleum ether, 1:3) gave methyl (2E)-3-( 2 '-amino- $5^{\prime}$-fluorophenyl)prop-2-enoate $(4 \mathrm{~d})(2.50 \mathrm{~g}, 81 \%)$ as a yellow solid. Mp $96-98{ }^{\circ} \mathrm{C}$ (lit. ${ }^{10} 93-95^{\circ} \mathrm{C}$ ); $R_{f}=0.28$ (diethyl ether/petroleum ether =1:1); ${ }^{1} \mathrm{H}$ NMR (400 MHz, $\left.\mathrm{CDCl}_{3}\right) \delta 3.80(\mathrm{~s}, 3 \mathrm{H}), 3.86(\mathrm{br} \mathrm{s}, 2 \mathrm{H}), 6.33(\mathrm{~d}, J=$ $15.8 \mathrm{~Hz}, 1 \mathrm{H}), 6.65\left(\mathrm{dd}, J=8.7,{ }^{4} J_{\mathrm{HF}}=4.8 \mathrm{~Hz}, 1 \mathrm{H}\right), 6.90(\mathrm{td}, J=8.7$, $2.9 \mathrm{~Hz}, 1 \mathrm{H}), 7.08\left(\mathrm{dd},{ }^{3} J_{\mathrm{HF}}=9.5, J=2.9 \mathrm{~Hz}, 1 \mathrm{H}\right), 7.76(\mathrm{dd}, J=15.8$, $\left.{ }^{5} J_{\mathrm{HF}}=1.1 \mathrm{~Hz}, 1 \mathrm{H}\right) ;{ }^{13} \mathrm{C} \mathrm{NMR}\left(101 \mathrm{MHz}, \mathrm{CDCl}_{3}\right) \delta 51.8\left(\mathrm{CH}_{3}\right), 113.4$ $\left(\mathrm{d},{ }^{2} J_{\mathrm{CF}}=22.7 \mathrm{~Hz}, \mathrm{CH}\right), 118.0\left(\mathrm{~d},{ }^{3} J_{\mathrm{CF}}=7.7 \mathrm{~Hz}, \mathrm{CH}\right), 118.3\left(\mathrm{~d},{ }^{2} J_{\mathrm{CF}}=\right.$ $23.0 \mathrm{~Hz}, \mathrm{CH}), 118.8(\mathrm{CH}), 120.8\left(\mathrm{~d},{ }^{3} J_{C F}=7.2 \mathrm{~Hz}, \mathrm{C}\right), 139.1\left(\mathrm{~d},{ }^{4} J_{C F}\right.$ $=2.2 \mathrm{~Hz}, \mathrm{CH}), 141.8(\mathrm{C}), 156.2\left(\mathrm{~d},{ }^{1} J_{\mathrm{CF}}=237.0 \mathrm{~Hz}, \mathrm{C}\right), 167.3(\mathrm{C})$; MS (ESI) $m / z 218\left(\mathrm{MNa}^{+}, 100\right), 169(25), 186$ (13), 164 (20).

Methyl (2E)-3-(2'-Amino-4'-fluorophenyl)prop-2-enoate (4e). ${ }^{17}$ The reaction was carried out as described for the synthesis of methyl (2E)-3-(2'-aminophenyl)prop-2-enoate (4a) using 5-fluoro2-iodoaniline $(3 \mathrm{e})(0.926 \mathrm{~g}, 3.90 \mathrm{mmol})$ and potassium carbonate $(1.08 \mathrm{~g}, 7.80 \mathrm{mmol})$. Purification by column chromatography (ethyl acetate/petroleum ether, 1:3) gave methyl (2E)-3-( $2^{\prime}$-amino- $4^{\prime}$ fluorophenyl)prop-2-enoate $(4 \mathrm{e})(0.639 \mathrm{~g}, 84 \%)$ as a yellow solid. Mp $107-109^{\circ} \mathrm{C} ; R_{f}=0.25$ (diethyl ether/petroleum ether $=1: 1$ ). Spectroscopic data were consistent with the literature. ${ }^{17}{ }^{1} \mathrm{H}$ NMR $\left(400 \mathrm{MHz}, \mathrm{CDCl}_{3}\right) \delta 3.80(\mathrm{~s}, 3 \mathrm{H}), 4.11(\mathrm{br} \mathrm{s}, 2 \mathrm{H}), 6.29(\mathrm{~d}, J=15.8$ $\mathrm{Hz}, 1 \mathrm{H}), 6.39\left(\mathrm{dd},{ }^{3} J_{\mathrm{HF}}=10.5, J=2.5 \mathrm{~Hz}, 1 \mathrm{H}\right), 6.47(\mathrm{td}, J=8.7,2.5$ $\mathrm{Hz}, 1 \mathrm{H}), 7.34\left(\mathrm{dd}, J=8.7,{ }^{4} J_{\mathrm{HF}}=6.4 \mathrm{~Hz}, 1 \mathrm{H}\right), 7.74(\mathrm{~d}, J=15.8 \mathrm{~Hz}$, $1 \mathrm{H}) ;{ }^{13} \mathrm{C}$ NMR $\left(101 \mathrm{MHz}, \mathrm{CDCl}_{3}\right) \delta 51.7\left(\mathrm{CH}_{3}\right), 102.9\left(\mathrm{~d},{ }^{2} J_{\mathrm{CF}}=\right.$ $24.8 \mathrm{~Hz}, \mathrm{CH}), 106.3\left(\mathrm{~d},{ }^{2} J_{\mathrm{CF}}=22.2 \mathrm{~Hz}, \mathrm{CH}\right), 116.0\left(\mathrm{~d},{ }^{4} J_{\mathrm{CF}}=2.4 \mathrm{~Hz}\right.$, C), $117.2(\mathrm{CH}), 130.0\left(\mathrm{~d},{ }^{3} J_{\mathrm{CF}}=10.6 \mathrm{~Hz}, \mathrm{CH}\right), 139.3(\mathrm{CH}), 147.4(\mathrm{~d}$, $\left.{ }^{3} J_{C F}=11.5 \mathrm{~Hz}, \mathrm{C}\right), 164.9\left(\mathrm{~d},{ }^{1} J_{\mathrm{CF}}=248.9 \mathrm{~Hz}, \mathrm{C}\right), 167.6(\mathrm{C})$; MS (ESI) $\mathrm{m} / z 218\left(\mathrm{MNa}^{+}, 100\right), 186$ (59), 164 (6).

Methyl (2E)-3-(2'-Amino-5'-chlorophenyl)prop-2-enoate (4f). ${ }^{10}$ The reaction was carried out as described for the synthesis of methyl (2E)-3-(2'-aminophenyl)prop-2-enoate (4a) using 4-chloro-2iodoaniline (3f) $(0.975 \mathrm{~g}, 3.90 \mathrm{mmol})$ and potassium carbonate $(1.08$ g, $7.80 \mathrm{mmol})$. The reaction mixture was stirred at $80{ }^{\circ} \mathrm{C}$ for $8 \mathrm{~h}$. Purification by column chromatography (ethyl acetate/petroleum ether = 1:3) gave methyl (2E)-3-(2'-amino-5' -chlorophenyl)prop-2enoate (4f) $(0.622 \mathrm{~g}, 76 \%)$ as a yellow solid. Mp 92-94 ${ }^{\circ} \mathrm{C} ; R_{f}=0.18$ (diethyl ether/petroleum ether $=1: 1)$. Spectroscopic data were consistent with the literature. ${ }^{10}{ }^{1} \mathrm{H}$ NMR $\left(400 \mathrm{MHz}, \mathrm{CDCl}_{3}\right) \delta 3.81$ (s, 3H), $3.97($ br s, $2 \mathrm{H}), 6.34(\mathrm{~d}, J=15.8 \mathrm{~Hz}, 1 \mathrm{H}), 6.64(\mathrm{~d}, J=8.6 \mathrm{~Hz}$, $1 \mathrm{H}), 7.12(\mathrm{dd}, J=8.6,2.4 \mathrm{~Hz}, 1 \mathrm{H}), 7.34(\mathrm{~d}, J=2.4 \mathrm{~Hz}, 1 \mathrm{H}), 7.73(\mathrm{~d}, J$ $=15.8 \mathrm{~Hz}, 1 \mathrm{H}) ;{ }^{13} \mathrm{C} \mathrm{NMR}\left(101 \mathrm{MHz}, \mathrm{CDCl}_{3}\right) \delta 51.8\left(\mathrm{CH}_{3}\right), 117.9$ $(\mathrm{CH}), 119.0(\mathrm{CH}), 121.1(\mathrm{C}), 123.7(\mathrm{C}), 127.3(\mathrm{CH}), 131.0(\mathrm{CH})$, 138.9 (CH), 144.0 (C), 167.3 (C); MS (ESI) $m / z 234\left(\mathrm{MNa}^{+}, 64\right)$, 202 (46), $186(100)$.

Methyl (2E)-3-(2'-[N-(p-Toluenesulfonyl)amino]phenyl)prop-2-enoate. ${ }^{18} p$-Toluenesulfonyl chloride $(2.50 \mathrm{~g}, 13.0 \mathrm{mmol})$ was added to a solution of methyl (2E)-3-(2'-aminophenyl)prop-2enoate $(4 \mathrm{a})(1.53 \mathrm{~g}, 8.70 \mathrm{mmol})$ in pyridine $(43 \mathrm{~mL})$ at $0{ }^{\circ} \mathrm{C}$. The reaction mixture was allowed to warm to room temperature and stirred for $1 \mathrm{~h}$. The reaction mixture was diluted with water $(50 \mathrm{~mL})$ and extracted with dichloromethane $(3 \times 50 \mathrm{~mL})$, washed with lithium chloride solution $(10 \mathrm{~mL})$ and brine $(10 \mathrm{~mL})$, dried $\left(\mathrm{MgSO}_{4}\right)$, filtered, and concentrated in vacuo. Flash column chromatography (diethyl ether/petroleum ether, 1:1) afforded methyl (2E)-3- $\left(2^{\prime}-[N-(p-\right.$ toluenesulfonyl)amino] phenyl)prop-2-enoate $(2.66 \mathrm{~g}, 93 \%)$ as a white solid. Mp $156-158{ }^{\circ} \mathrm{C}$ (lit. ${ }^{18} 160-162{ }^{\circ} \mathrm{C}$ ); $R_{f}=0.13$ (diethyl ether/petroleum ether $=1: 1) ;{ }^{1} \mathrm{H}$ NMR $\left(400 \mathrm{MHz}, \mathrm{CDCl}_{3}\right) \delta 2.35(\mathrm{~s}$, $3 \mathrm{H}), 3.77(\mathrm{~s}, 3 \mathrm{H}), 6.11(\mathrm{~d}, J=15.8 \mathrm{~Hz}, 1 \mathrm{H}), 7.17(\mathrm{~d}, J=8.2 \mathrm{~Hz}, 2 \mathrm{H})$, $7.20-7.27(\mathrm{~m}, 2 \mathrm{H}), 7.34(\mathrm{td}, J=8.0,1.5 \mathrm{~Hz}, 1 \mathrm{H}), 7.40(\mathrm{dd}, J=8.0$, $1.2 \mathrm{~Hz}, 1 \mathrm{H}), 7.45$ (dd, $J=8.0,1.5 \mathrm{~Hz}, 1 \mathrm{H}), 7.54(\mathrm{~d}, J=8.2 \mathrm{~Hz}, 2 \mathrm{H})$, $7.62(\mathrm{~d}, J=15.8 \mathrm{~Hz}, 1 \mathrm{H}) ;{ }^{13} \mathrm{C} \mathrm{NMR}\left(101 \mathrm{MHz}, \mathrm{CDCl}_{3}\right) \delta 21.5$ $\left(\mathrm{CH}_{3}\right), 51.9\left(\mathrm{CH}_{3}\right), 120.1(\mathrm{CH}), 127.1(\mathrm{CH}), 127.2(\mathrm{CH}), 127.3(2 \times$ $\mathrm{CH}), 127.6(\mathrm{CH}), 129.6(2 \times \mathrm{CH}), 130.6(\mathrm{C}), 130.9(\mathrm{CH}), 134.8$ (C), 135.9 (C), $139.3(\mathrm{CH}), 143.9$ (C), 167.0 (C); MS (ESI) $m / z 354$ $\left(\mathrm{MNa}^{+}, 100\right), 233(8)$

Methyl (2E)-3-(5'-Methyl-2'-[N-(p-toluenesulfonyl)amino]phenyl)prop-2-enoate. ${ }^{19}$ The reaction was carried out as described for the synthesis of methyl $(2 E)-3-\left(2^{\prime}-[N-(p\right.$-toluenesulfonyl $)$ amino $]$ phenyl)prop-2-enoate using methyl (2E)-3-(2'-amino-5'-methylphenyl)prop-2-enoate $(4 \mathbf{b})(1.50 \mathrm{~g}, 7.84 \mathrm{mmol})$. Purification by column chromatography (ethyl acetate/petroleum ether $=1: 4)$ gave methyl (2E)-3-(5'-methyl-2' - $N$-( $p$-toluenesulfonyl)amino $]$ phenyl)prop-2-enoate $(2.68 \mathrm{~g}, 99 \%)$ as a white solid. Mp $164-166{ }^{\circ} \mathrm{C}$ (lit. $\left.{ }^{19} 160-162{ }^{\circ} \mathrm{C}\right) ; R_{f}=0.20$ (diethyl ether/petroleum ether = 1:1); ${ }^{1} \mathrm{H}$ NMR (400 MHz, $\mathrm{CDCl}_{3}$ ) $\delta 2.32(\mathrm{~s}, 3 \mathrm{H}), 2.35$ (s, 3H), 3.77 (s, $3 \mathrm{H}), 6.10(\mathrm{~d}, J=15.9 \mathrm{~Hz}, 1 \mathrm{H}), 6.99(\mathrm{br} \mathrm{s}, 1 \mathrm{H}), 7.12-7.19(\mathrm{~m}, 3 \mathrm{H})$, 7.23-7.26 (m, 2H), 7.50-7.57 (m, 3H); ${ }^{13} \mathrm{C}$ NMR (101 MHz, $\left.\mathrm{CDCl}_{3}\right) \delta 21.0\left(\mathrm{CH}_{3}\right), 21.5\left(\mathrm{CH}_{3}\right), 51.8\left(\mathrm{CH}_{3}\right), 119.7(\mathrm{CH}), 127.3(2$ $\times \mathrm{CH}), 127.4(\mathrm{CH}), 128.0(\mathrm{CH}), 129.6(2 \times \mathrm{CH}), 130.7(\mathrm{C}), 131.8$ (CH), $132.1(\mathrm{C}), 135.9(\mathrm{C}), 137.4(\mathrm{C}), 139.4(\mathrm{CH}), 143.8(\mathrm{C}), 167.0$ (C); MS (ESI) $m / z 368\left(\mathrm{MNa}^{+}, 100\right)$.

Methyl (2E)-3-(5'-Methoxy-2'-[N-(p-toluenesulfonyl)amino]phenyl)prop-2-enoate. The reaction was carried out as described for the synthesis of methyl $(2 E)-3-\left(2^{\prime}-[N-(p\right.$-toluenesulfonyl $)$ amino $]$ phenyl)prop-2-enoate using methyl (2E)-3-(2'-amino- $5^{\prime}$-methoxyphenyl)prop-2-enoate $(4 \mathrm{c})(0.014 \mathrm{~g}, 0.070 \mathrm{mmol})$. Purification by column chromatography (ethyl acetate/petroleum ether $=1: 4$ ) gave methyl (2E)-3-(5'-methoxy-2'-[N-(p-toluenesulfonyl)amino $]$ phenyl)prop-2-enoate $(0.023 \mathrm{~g}, 93 \%)$ as a white solid. Mp $162-164{ }^{\circ} \mathrm{C} ; R_{f}=$ 0.23 (petroleum ether/ethyl acetate $=2: 1$ ); IR (neat) 3256, 3023, $1701,1637,1495,1214,1325,1161,750 \mathrm{~cm}^{-1}$; ${ }^{1} \mathrm{H}$ NMR $(500 \mathrm{MHz}$, $\left.\mathrm{CDCl}_{3}\right) \delta 2.37(\mathrm{~s}, 3 \mathrm{H}), 3.78(\mathrm{~s}, 3 \mathrm{H}), 3.81(\mathrm{~s}, 3 \mathrm{H}), 6.09(\mathrm{~d}, J=15.9 \mathrm{~Hz}$, $1 \mathrm{H}), 6.53$ (br s, $1 \mathrm{H}), 6.89$ (dd, $J=8.8,2.9 \mathrm{~Hz}, 1 \mathrm{H}), 6.95(\mathrm{~d}, J=2.9$ $\mathrm{Hz}, 1 \mathrm{H}), 7.19$ (d, $J=8.2 \mathrm{~Hz}, 2 \mathrm{H}), 7.23(\mathrm{~d}, J=8.8 \mathrm{~Hz}, 1 \mathrm{H}), 7.46(\mathrm{~d}, J$ $=15.9 \mathrm{~Hz}, 1 \mathrm{H}), 7.52(\mathrm{~d}, J=8.2 \mathrm{~Hz}, 2 \mathrm{H}) ;{ }^{13} \mathrm{C} \operatorname{NMR}(101 \mathrm{MHz}$, $\left.\mathrm{CDCl}_{3}\right) \delta 21.5\left(\mathrm{CH}_{3}\right), 51.8\left(\mathrm{CH}_{3}\right), 55.5\left(\mathrm{CH}_{3}\right), 111.4(\mathrm{CH}), 116.7$ $(\mathrm{CH}), 120.1(\mathrm{CH}), 127.3(\mathrm{C}), 127.4(2 \times \mathrm{CH}), 129.6(2 \times \mathrm{CH})$, $130.6(\mathrm{CH}), 133.1(\mathrm{C}), 135.8(\mathrm{C}), 139.2(\mathrm{CH}), 143.9(\mathrm{C}), 158.9(\mathrm{C})$, 166.7 (C); MS (ESI) $m / z 384\left(\mathrm{MNa}^{+}, 100\right)$; HRMS (ESI) calcd for $\mathrm{C}_{18} \mathrm{H}_{19} \mathrm{NNaO}_{5} \mathrm{~S}\left(\mathrm{MNa}^{+}\right)$, 384.0876; found, 384.0864.

Methyl (2E)-3-(5'-Fluoro-2'-[N-(p-toluenesulfonyl)amino]phenyl)prop-2-enoate. ${ }^{19}$ The reaction was carried out as described for the synthesis of methyl $(2 E)-3-\left(2^{\prime}-[N-(p\right.$-toluenesulfonyl $)$ amino $]-$ phenyl)prop-2-enoate using methyl (2E)-3-(2'-amino-5'-fluorophenyl)prop-2-enoate $(4 \mathrm{~d})(2.50 \mathrm{~g}, 13.0 \mathrm{mmol})$. Purification by column chromatography (ethyl acetate/petroleum ether $=1: 5$ ) gave methyl (2E)-3-(5'-fluoro-2'-[N-( -toluenesulfonyl)amino $]$ phenyl)prop-2-enoate $(3.94 \mathrm{~g}, 88 \%)$ as a white solid. Mp $156-158{ }^{\circ} \mathrm{C}$ (lit. $\left.{ }^{19} 156-158{ }^{\circ} \mathrm{C}\right) ; R_{f}=0.13$ (diethyl ether/petroleum ether = 1:1); ${ }^{1} \mathrm{H}$ NMR $\left(400 \mathrm{MHz}, \mathrm{CDCl}_{3}\right) \delta 2.36(\mathrm{~s}, 3 \mathrm{H}), 3.78(\mathrm{~s}, 3 \mathrm{H}), 6.07(\mathrm{~d}, J=$ $15.8 \mathrm{~Hz}, 1 \mathrm{H}), 6.96(\mathrm{br} \mathrm{s}, 1 \mathrm{H}), 7.06\left(\mathrm{ddd}, J=8.8,{ }^{3} J_{\mathrm{HF}}=7.7, J=2.9 \mathrm{~Hz}\right.$, $1 \mathrm{H}), 7.14\left(\mathrm{dd},{ }^{3} J_{\mathrm{HF}}=9.2, J=2.9 \mathrm{~Hz}, 1 \mathrm{H}\right), 7.19(\mathrm{~d}, J=8.1 \mathrm{~Hz}, 2 \mathrm{H})$, $7.35\left(\mathrm{dd}, J=8.8,{ }^{4} J_{H F}=5.2 \mathrm{~Hz}, 1 \mathrm{H}\right), 7.50\left(\mathrm{dd}, J=15.8,{ }^{5} J_{\mathrm{HF}}=1.5 \mathrm{~Hz}\right.$, $1 \mathrm{H}), 7.52(\mathrm{~d}, J=8.1 \mathrm{~Hz}, 2 \mathrm{H}) ;{ }^{13} \mathrm{C}$ NMR $\left(101 \mathrm{MHz}, \mathrm{CDCl}_{3}\right) \delta 21.5$ $\left(\mathrm{CH}_{3}\right), 52.0\left(\mathrm{CH}_{3}\right), 113.3\left(\mathrm{~d},{ }^{2} J_{\mathrm{CF}}=23.5 \mathrm{~Hz}, \mathrm{CH}\right), 117.9\left(\mathrm{~d},{ }^{2} J_{\mathrm{CF}}=\right.$ $22.7 \mathrm{~Hz}, \mathrm{CH}), 121.2(\mathrm{CH}), 127.3(2 \times \mathrm{CH}), 129.7(2 \times \mathrm{CH}), 130.6$ $\left(\mathrm{d},{ }^{4} J_{\mathrm{CF}}=2.9 \mathrm{~Hz}, \mathrm{C}\right), 130.7\left(\mathrm{~d},{ }^{3} J_{\mathrm{CF}}=8.8 \mathrm{~Hz}, \mathrm{CH}\right), 133.4\left(\mathrm{~d},{ }^{3} J_{\mathrm{CF}}=8.4\right.$ 
$\mathrm{Hz}, \mathrm{C}), 135.6$ (C), $138.2\left(\mathrm{~d},{ }^{4} J_{\mathrm{CF}}=2.2 \mathrm{~Hz}, \mathrm{CH}\right), 144.2(\mathrm{C}), 161.5$ (d, $\left.{ }^{1} J_{C F}=248.4 \mathrm{~Hz}, \mathrm{C}\right), 166.5$ (C); MS (ESI) $m / z 372\left(\mathrm{MNa}^{+}, 100\right)$.

Methyl (2E)-3-(4'-Fluoro-2'-[N-(p-toluenesulfonyl)amino]phenyl)prop-2-enoate. ${ }^{17}$ The reaction was carried out as described for the synthesis of methyl (2E)-3-( $2^{\prime}-[N-(p$-toluenesulfonyl)amino $]$ phenyl)prop-2-enoate using methyl (2E)-3-(2'-amino-4'-fluorophenyl)prop-2-enoate $(4 \mathrm{e})(0.620 \mathrm{~g}, 3.20 \mathrm{mmol})$. Purification by column chromatography (ethyl acetate/petroleum ether $=1: 5$ ) gave methyl (2E)-3-(4'-fluoro- $2^{\prime}$-[ $N$-( $p$-toluenesulfonyl)amino $]$ phenyl)prop-2-enoate $(1.08 \mathrm{~g}, 97 \%)$ as a yellow solid. Mp $157-159{ }^{\circ} \mathrm{C} ; R_{f}$ $=0.13$ (diethyl ether/petroleum ether $=1: 1)$. Spectroscopic data were consistent with the literature. ${ }^{17}{ }^{1} \mathrm{H} \mathrm{NMR}\left(400 \mathrm{MHz}, \mathrm{CDCl}_{3}\right) \delta 2.38$ $(\mathrm{s}, 3 \mathrm{H}), 3.79(\mathrm{~s}, 3 \mathrm{H}), 6.11(\mathrm{~d}, J=15.8 \mathrm{~Hz}, 1 \mathrm{H}), 6.92(\mathrm{td}, J=8.7,2.6$ $\mathrm{Hz}, 1 \mathrm{H}), 7.00$ (br s, $1 \mathrm{H}), 7.20-7.26(\mathrm{~m}, 3 \mathrm{H}), 7.41\left(\mathrm{dd}, J=8.7,{ }^{4} J_{\mathrm{HF}}=\right.$ $6.1 \mathrm{~Hz}, 1 \mathrm{H}), 7.48(\mathrm{~d}, J=15.8 \mathrm{~Hz}, 1 \mathrm{H}), 7.63(\mathrm{~d}, J=8.3 \mathrm{~Hz}, 2 \mathrm{H}) ;{ }^{13} \mathrm{C}$ $\operatorname{NMR}\left(101 \mathrm{MHz}, \mathrm{CDCl}_{3}\right) \delta 21.5\left(\mathrm{CH}_{3}\right), 51.9\left(\mathrm{CH}_{3}\right), 112.8\left(\mathrm{~d},{ }^{2} J_{\mathrm{CF}}=\right.$ $24.9 \mathrm{~Hz}, \mathrm{CH}), 114.1\left(\mathrm{~d},{ }^{2} J_{\mathrm{CF}}=21.8 \mathrm{~Hz}, \mathrm{CH}\right), 120.5(\mathrm{CH}), 125.1(\mathrm{~d}$, $\left.{ }^{4} J_{C F}=3.4 \mathrm{~Hz}, \mathrm{C}\right), 127.3(2 \times \mathrm{CH}), 128.9\left(\mathrm{~d},{ }^{3} J_{C F}=9.5 \mathrm{~Hz}, \mathrm{CH}\right), 129.9$ $(2 \times \mathrm{CH}), 135.7(\mathrm{C}), 136.5\left(\mathrm{~d},{ }^{3} J_{\mathrm{CF}}=10.8 \mathrm{~Hz}, \mathrm{C}\right), 137.8(\mathrm{CH}), 144.4$ (C), 163.8 (d, ${ }^{1} J_{C F}=251.8 \mathrm{~Hz}, \mathrm{C}$ ), 166.7 (C); MS (ESI) $\mathrm{m} / z 372$ $\left(\mathrm{MNa}^{+}, 100\right), 363(37)$.

Methyl (2E)-3-(5'-Chloro-2'-[N-( $p$-toluenesulfonyl)amino]phenyl)prop-2-enoate. ${ }^{19}$ The reaction was carried out as described for the synthesis of methyl $(2 E)-3-\left(2^{\prime}-[N-(p\right.$-toluenesulfonyl $)$ amino $]-$ phenyl)prop-2-enoate using methyl (2E)-3-(2'-amino- $5^{\prime}$ chlorophenyl)prop-2-enoate $(4 \mathrm{f})(0.406 \mathrm{~g}, 1.90 \mathrm{mmol})$. The reaction mixture was stirred at room temperature for $18 \mathrm{~h}$. Purification by column chromatography (ethyl acetate/petroleum ether $=1: 5$ ) gave methyl (2E)-3-(5'-chloro-2'-[N-( $p$-toluenesulfonyl)amino $]$ phenyl)prop-2-enoate $(0.638 \mathrm{~g}, 91 \%)$ as a yellow solid. Mp $152-154{ }^{\circ} \mathrm{C}$ (lit. ${ }^{19} 149-151^{\circ} \mathrm{C}$ ); $R_{f}=0.43$ (petroleum ether/ethyl acetate $=2: 1$ ); ${ }^{1} \mathrm{H}$ NMR $\left(400 \mathrm{MHz}, \mathrm{CDCl}_{3}\right) \delta 2.36(\mathrm{~s}, 3 \mathrm{H}), 3.78(\mathrm{~s}, 3 \mathrm{H}), 6.09(\mathrm{~d}, J=$ $15.8 \mathrm{~Hz}, 1 \mathrm{H}), 7.19(\mathrm{~d}, J=8.0 \mathrm{~Hz}, 2 \mathrm{H}), 7.28(\mathrm{br} \mathrm{s}, 1 \mathrm{H}), 7.31(\mathrm{dd}, J=$ 8.6, $2.4 \mathrm{~Hz}, 1 \mathrm{H}), 7.36(\mathrm{~d}, J=8.6 \mathrm{~Hz}, 1 \mathrm{H}), 7.40(\mathrm{~d}, J=2.4 \mathrm{~Hz}, 1 \mathrm{H})$, $7.50-7.56(\mathrm{~m}, 3 \mathrm{H}) ;{ }^{13} \mathrm{C}$ NMR $\left(101 \mathrm{MHz}, \mathrm{CDCl}_{3}\right) \delta 21.5\left(\mathrm{CH}_{3}\right), 52.1$ $\left(\mathrm{CH}_{3}\right), 121.3(\mathrm{CH}), 126.9(\mathrm{CH}), 127.3(2 \times \mathrm{CH}), 129.1(\mathrm{CH}), 129.8$ $(2 \times \mathrm{CH}), 130.8(\mathrm{CH}), 132.2(\mathrm{C}), 133.1(\mathrm{C}), 133.3(\mathrm{C}), 135.6(\mathrm{C})$, $138.1(\mathrm{CH}), 144.2(\mathrm{C}), 166.7$ (C); MS (ESI) $m / z 388\left(\mathrm{MNa}^{+}, 100\right)$.

Methyl (2E)-3-(2'-[N-Allyl- $N$-( $p$-toluenesulfonyl)amino]phenyl)prop-2-enoate (5a). Allyl bromide $(0.830 \mathrm{~mL}, 9.60$ $\mathrm{mmol})$ was added to a stirred solution of methyl $(2 E)-3-\left(2^{\prime}-[N-(p-\right.$ toluenesulfonyl)amino]phenyl)prop-2-enoate $(2.66 \mathrm{~g}, 8.00 \mathrm{mmol})$ and potassium carbonate $(2.21 \mathrm{~g}, 16.0 \mathrm{mmol})$ in $N, N^{\prime}$-dimethylformamide $(50 \mathrm{~mL})$. The reaction mixture was heated to $70{ }^{\circ} \mathrm{C}$ for $1 \mathrm{~h}$. The reaction mixture was cooled to room temperature, diluted with $5 \%$ aqueous lithium chloride solution $(20 \mathrm{~mL})$, and extracted with diethyl ether $(50 \mathrm{~mL})$. The organic layer was washed with $5 \%$ aqueous lithium chloride solution $(3 \times 10 \mathrm{~mL})$, brine $(10 \mathrm{~mL})$, dried $\left(\mathrm{MgSO}_{4}\right)$, filtered, and concentrated in vacuo. Purification by column chromatography (diethyl ether/petroleum ether $=1: 1$ ) gave methyl (2E)-3-(2'-[N-allyl- $N$-( $p$-toluenesulfonyl) amino $]$ phenyl)prop-2enoate (5a) $(2.98 \mathrm{~g}, 100 \%)$ as a white solid. $\mathrm{Mp} 104-106{ }^{\circ} \mathrm{C}$; $R_{f}=$ 0.38 (diethyl ether/petroleum ether $=1: 1$ ); IR (neat) 2951, 1716, 1636, 1436, 1319, 1164, $763 \mathrm{~cm}^{-1}$; ${ }^{1} \mathrm{H}$ NMR (400 MHz, $\left.\mathrm{CDCl}_{3}\right) \delta$ $2.42(\mathrm{~s}, 3 \mathrm{H}), 3.79(\mathrm{~s}, 3 \mathrm{H}), 4.02(\mathrm{br} \mathrm{s}, 1 \mathrm{H}), 4.27(\mathrm{br} \mathrm{s}, 1 \mathrm{H}), 4.93-5.02$ $(\mathrm{m}, 2 \mathrm{H}), 5.74(\mathrm{ddt}, J=17.0,10.0,6.8 \mathrm{~Hz}, 1 \mathrm{H}), 6.33(\mathrm{~d}, J=16.1 \mathrm{~Hz}$, $1 \mathrm{H}), 6.84(\mathrm{dd}, J=7.8,1.1 \mathrm{~Hz}, 1 \mathrm{H}), 7.24-7.35(\mathrm{~m}, 4 \mathrm{H}), 7.56(\mathrm{~d}, J=$ $8.2 \mathrm{~Hz}, 2 \mathrm{H}), 7.64(\mathrm{dd}, J=7.8,1.5 \mathrm{~Hz}, 1 \mathrm{H}), 7.86(\mathrm{~d}, J=16.1 \mathrm{~Hz}, 1 \mathrm{H})$; ${ }^{13} \mathrm{C} \mathrm{NMR}\left(101 \mathrm{MHz}, \mathrm{CDCl}_{3}\right) \delta 21.5\left(\mathrm{CH}_{3}\right), 51.7\left(\mathrm{CH}_{3}\right), 54.9\left(\mathrm{CH}_{2}\right)$, $119.7(\mathrm{CH}), 119.7\left(\mathrm{CH}_{2}\right), 127.1(\mathrm{CH}), 128.0(2 \times \mathrm{CH}), 128.8(\mathrm{CH})$, $129.6(2 \times \mathrm{CH}), 129.9(\mathrm{CH}), 130.3(\mathrm{CH}), 132.1(\mathrm{CH}), 135.6(\mathrm{C})$, 135.6 (C), $138.3(\mathrm{C}), 140.3(\mathrm{CH}), 143.8$ (C), 166.9 (C); MS (ESI) $m / z 394\left(\mathrm{MNa}^{+}, 100\right)$; HRMS (ESI) calcd for $\mathrm{C}_{20} \mathrm{H}_{21} \mathrm{NNaO}_{4} \mathrm{~S}$ $\left(\mathrm{MNa}^{+}\right)$, 394.1083; found, 394.1067.

Methyl (2E)-3-(2'-[N-Allyl- $N$-( $p$-toluenesulfonyl)amino]-5'methylphenyl)prop-2-enoate (5b). The reaction was carried out as described for the synthesis of methyl (2E)-3-( $2^{\prime}-[N-$ allyl- $N-(p-$ toluenesulfonyl)amino]phenyl)prop-2-enoate (5a) using methyl $(2 E)$ 3 -(5'-methyl-2'-[N-( $p$-toluenesulfonyl)amino $]$ phenyl) prop-2-enoate $(2.00 \mathrm{~g}, 5.79 \mathrm{mmol})$ and a reaction time of $3 \mathrm{~h}$. Purification by column chromatography (ethyl acetate/petroleum ether $=1: 5$ ) gave methyl (2E)-3-(2'-[N-allyl- $N$-( $p$-toluenesulfonyl)amino]-5' -methylphenyl)prop-2-enoate $(5 \mathbf{b})(2.03 \mathrm{~g}, 91 \%)$ as a white solid. Mp $118-120^{\circ} \mathrm{C} ; R_{f}$ $=0.25$ (diethyl ether/petroleum ether $=1: 1$ ); IR (neat) 2950, 1717, $1639,1435,1347,1160,759 \mathrm{~cm}^{-1} ;{ }^{1} \mathrm{H}$ NMR (400 MHz, $\left.\mathrm{CDCl}_{3}\right) \delta$ $2.34(\mathrm{~s}, 3 \mathrm{H}), 2.42(\mathrm{~s}, 3 \mathrm{H}), 3.78(\mathrm{~s}, 3 \mathrm{H}), 3.99(\mathrm{br} \mathrm{s}, 1 \mathrm{H}), 4.26(\mathrm{br} \mathrm{s}$, $1 \mathrm{H}), 4.93-5.02(\mathrm{~m}, 2 \mathrm{H}), 5.74$ (ddt, $J=17.0,10.1,6.8 \mathrm{~Hz}, 1 \mathrm{H}), 6.31$ $(\mathrm{d}, J=16.1 \mathrm{~Hz}, 1 \mathrm{H}), 6.71(\mathrm{~d}, J=8.1 \mathrm{~Hz}, 1 \mathrm{H}), 7.08(\mathrm{dd}, J=8.1,1.6$ $\mathrm{Hz}, 1 \mathrm{H}), 7.26(\mathrm{~d}, J=8.2 \mathrm{~Hz}, 2 \mathrm{H}), 7.43(\mathrm{~d}, J=1.6 \mathrm{~Hz}, 1 \mathrm{H}), 7.56$ (d, $J$ $=8.2 \mathrm{~Hz}, 2 \mathrm{H}), 7.82(\mathrm{~d}, J=16.1 \mathrm{~Hz}, 1 \mathrm{H}) ;{ }^{13} \mathrm{C} \mathrm{NMR}(101 \mathrm{MHz}$, $\left.\mathrm{CDCl}_{3}\right) \delta 21.2\left(\mathrm{CH}_{3}\right), 21.5\left(\mathrm{CH}_{3}\right), 51.7\left(\mathrm{CH}_{3}\right), 54.9\left(\mathrm{CH}_{2}\right), 119.4$ $(\mathrm{CH}), 119.6\left(\mathrm{CH}_{2}\right), 127.6(\mathrm{CH}), 128.0(2 \times \mathrm{CH}), 129.5(2 \times \mathrm{CH})$, $129.6(\mathrm{CH}), 131.3(\mathrm{CH}), 132.3(\mathrm{CH}), 135.2(\mathrm{C}), 135.7(\mathrm{C}), 135.7$ (C), 138.7 (C), 140.5 (CH), 143.7 (C), 167.0 (C); MS (ESI) $m / z 408$ $\left(\mathrm{MNa}^{+}, 100\right)$; HRMS (ESI) calcd for $\mathrm{C}_{21} \mathrm{H}_{23} \mathrm{NNaO}_{4} \mathrm{~S}\left(\mathrm{MNa}^{+}\right)$, 408.1240; found, 408.1220.

Methyl (2E)-3-(2'-[N-Allyl- $N$-( $p$-toluenesulfonyl)amino]-5'methoxyphenyl)prop-2-enoate $(5 \mathrm{c})$. The reaction was carried out as described for the synthesis of methyl $(2 E)-3-\left(2^{\prime}-[N\right.$-allyl- $N-(p-$ toluenesulfonyl)amino]phenyl)prop-2-enoate (5a) using methyl $(2 E)$ 3-(5'-methoxy-2' - $[N$-( $p$-toluenesulfonyl)amino]phenyl)prop-2-enoate $(0.145 \mathrm{~g}, 0.400 \mathrm{mmol})$ and a reaction time of $2 \mathrm{~h}$. Purification by column chromatography (ethyl acetate/petroleum ether $=1: 5$ ) gave methyl (2E)-3-(2'-[N-allyl- $N$-( $p$-toluenesulfonyl)amino $]-5^{\prime}$-methoxyphenyl)prop-2-enoate (5c) (0.149 g, 92\%) as a white solid. Mp 153-155 ${ }^{\circ} \mathrm{C} ; R_{f}=0.40$ (petroleum ether/ethyl acetate $=2: 1$ ); IR (neat) 3022, 1709, 1642, 1495, 1289, 1215, 1163, $751 \mathrm{~cm}^{-1}$; ${ }^{1} \mathrm{H}$ NMR $\left(400 \mathrm{MHz}, \mathrm{CDCl}_{3}\right) \delta 2.42(\mathrm{~s}, 3 \mathrm{H}), 3.79(\mathrm{~s}, 3 \mathrm{H}), 3.81(\mathrm{~s}, 3 \mathrm{H}), 3.96-$ $4.01(\mathrm{~m}, 1 \mathrm{H}), 4.24-4.29(\mathrm{~m}, 1 \mathrm{H}), 4.93-5.03(\mathrm{~m}, 2 \mathrm{H}), 5.74(\mathrm{ddt}, J=$ $16.9,10.1,6.8 \mathrm{~Hz}, 1 \mathrm{H}), 6.29(\mathrm{~d}, J=16.1 \mathrm{~Hz}, 1 \mathrm{H}), 6.74(\mathrm{~d}, J=8.8 \mathrm{~Hz}$, $1 \mathrm{H}), 6.81(\mathrm{dd}, J=8.8,2.8 \mathrm{~Hz}, 1 \mathrm{H}), 7.09(\mathrm{~d}, J=2.8 \mathrm{~Hz}, 1 \mathrm{H}), 7.26(\mathrm{~d}, J$ $=8.2 \mathrm{~Hz}, 2 \mathrm{H}), 7.56(\mathrm{~d}, J=8.2 \mathrm{~Hz}, 2 \mathrm{H}), 7.79(\mathrm{~d}, J=16.1 \mathrm{~Hz}, 1 \mathrm{H}) ;{ }^{13} \mathrm{C}$ NMR $\left(101 \mathrm{MHz}, \mathrm{CDCl}{ }_{3}\right) \delta 21.5\left(\mathrm{CH}_{3}\right), 51.7\left(\mathrm{CH}_{3}\right), 55.0\left(\mathrm{CH}_{2}\right)$, $55.5\left(\mathrm{CH}_{3}\right), 111.2(\mathrm{CH}), 116.4(\mathrm{CH}), 119.7\left(\mathrm{CH}_{2}\right), 119.8(\mathrm{CH})$, $128.0(2 \times \mathrm{CH}), 129.6(2 \times \mathrm{CH}), 131.0(\mathrm{CH}), 132.3(\mathrm{CH}), 135.7$ (C), 136.6 (C), $140.4(\mathrm{CH}), 143.7(2 \times \mathrm{C}), 159.3$ (C), 166.8 (C); MS (ESI) $m / z 424\left(\mathrm{MNa}^{+}, 100\right)$; HRMS (ESI) calcd for $\mathrm{C}_{21} \mathrm{H}_{23} \mathrm{NNaO}_{5} \mathrm{~S}$ $\left(\mathrm{MNa}^{+}\right), 424.1189$; found, 424.1176 .

Methyl (2E)-3-(2'-[N-Allyl- $N$-( $p$-toluenesulfonyl)amino]-5'fluorophenyl)prop-2-enoate $(5 d)$. The reaction was carried out as described for the synthesis of methyl (2E)-3- $\left(2^{\prime}-[N\right.$-allyl- $N-(p-$ toluenesulfonyl)amino]phenyl)prop-2-enoate (5a) using methyl (2E)3 -(5'-fluoro-2'-[N-( $p$-toluenesulfonyl)amino $]$ phenyl)prop-2-enoate $(3.74 \mathrm{~g}, 11.0 \mathrm{mmol})$. Purification by column chromatography (ethyl acetate/petroleum ether $=1: 7)$ gave methyl $(2 E)-3-\left(2^{\prime}-[N\right.$-allyl- $N-(p-$ toluenesulfonyl)amino]-5'-fluorophenyl)prop-2-enoate (5d) (3.50 g, $84 \%$ ) as a white solid. Mp $108-110{ }^{\circ} \mathrm{C} ; R_{f}=0.43$ (diethyl ether/ petroleum ether =1:1); IR (neat) 2951, 1718, 1650, 1488, 1323, 1275, 1160, 862, $728 \mathrm{~cm}^{-1}$; ${ }^{1} \mathrm{H}$ NMR $\left(400 \mathrm{MHz}, \mathrm{CDCl}_{3}\right) \delta 2.40(\mathrm{~s}, 3 \mathrm{H})$, 3.77 (s, 3H), 3.95 (br s, $1 \mathrm{H}), 4.25$ (br s, $1 \mathrm{H}), 4.95$ (dd, $J=17.0,1.2$ $\mathrm{Hz}, 1 \mathrm{H}), 5.00$ (dd, $J=10.1,1.2 \mathrm{~Hz}, 1 \mathrm{H}), 5.71$ (ddt, $J=17.0,10.1,6.8$ $\mathrm{Hz}, 1 \mathrm{H}), 6.28(\mathrm{~d}, J=16.1 \mathrm{~Hz}, 1 \mathrm{H}), 6.80\left(\mathrm{dd}, J=8.8,{ }^{4} J_{\mathrm{HF}}=5.3 \mathrm{~Hz}\right.$, $1 \mathrm{H}), 6.95\left(\mathrm{ddd}, J=8.8,{ }^{3} J_{\mathrm{HF}}=7.6, J=2.9 \mathrm{~Hz}, 1 \mathrm{H}\right), 7.26(\mathrm{~d}, J=8.2 \mathrm{~Hz}$, $2 \mathrm{H}), 7.28\left(\mathrm{dd},{ }^{3} J_{\mathrm{HF}}=9.4, J=2.9 \mathrm{~Hz}, 1 \mathrm{H}\right), 7.54(\mathrm{~d}, J=8.2 \mathrm{~Hz}, 2 \mathrm{H})$, $7.76\left(\mathrm{dd}, J=16.1,{ }^{5} \mathrm{HF}_{\mathrm{HF}}=1.6 \mathrm{~Hz}, 1 \mathrm{H}\right) ;{ }^{13} \mathrm{C} \mathrm{NMR}\left(101 \mathrm{MHz}, \mathrm{CDCl}_{3}\right) \delta$ $21.5\left(\mathrm{CH}_{3}\right), 51.8\left(\mathrm{CH}_{3}\right), 55.0\left(\mathrm{CH}_{2}\right), 113.5\left(\mathrm{~d},{ }^{2} J_{\mathrm{CF}}=23.4 \mathrm{~Hz}, \mathrm{CH}\right)$, $117.4\left(\mathrm{~d},{ }^{2} J_{\mathrm{CF}}=23.0 \mathrm{~Hz}, \mathrm{CH}\right), 120.1\left(\mathrm{CH}_{2}\right), 120.9(\mathrm{CH}), 127.9(2 \times$ $\mathrm{CH}), 129.7(2 \times \mathrm{CH}), 131.8\left(\mathrm{~d},{ }^{3} J_{\mathrm{CF}}=8.9 \mathrm{~Hz}, \mathrm{CH}\right), 131.9(\mathrm{CH})$, $134.2\left(\mathrm{~d},{ }^{4} J_{\mathrm{CF}}=3.1 \mathrm{~Hz}, \mathrm{C}\right), 135.3(\mathrm{C}), 137.8\left(\mathrm{~d},{ }^{3} J_{\mathrm{CF}}=8.5 \mathrm{~Hz}, \mathrm{C}\right)$, $139.2\left(\mathrm{~d},{ }^{4} J_{\mathrm{CF}}=2.0 \mathrm{~Hz}, \mathrm{CH}\right), 144.0(\mathrm{C}), 162.0\left(\mathrm{~d},{ }^{1} J_{\mathrm{CF}}=249.4 \mathrm{~Hz}, \mathrm{C}\right)$, 166.5 (C); MS (ESI) $m / z 412\left(\mathrm{MNa}^{+}, 100\right)$; HRMS (ESI) calcd for $\mathrm{C}_{20} \mathrm{H}_{20} \mathrm{FNNaO}_{4} \mathrm{~S}\left(\mathrm{MNa}^{+}\right)$, 412.0989; found, 412.0969.

Methyl (2E)-3-(2'-[N-Allyl- $N$-(p-toluenesulfonyl)amino]-4' fluorophenyl)prop-2-enoate $(5 \mathrm{e})$. The reaction was carried out as described for the synthesis of methyl (2E)-3- $\left(2^{\prime}-[N\right.$-allyl- $N-(p-$ toluenesulfonyl)amino]phenyl)prop-2-enoate (5a) using methyl (2E)3-(4'-fluoro-2' $-[N$ - $(p$-toluenesulfonyl)amino $]$ phenyl) prop-2-enoate $(1.07 \mathrm{~g}, 3.00 \mathrm{mmol})$. Purification by column chromatography (ethyl acetate/petroleum ether $=1: 7)$ gave methyl $(2 E)-3-\left(2^{\prime}-[N\right.$-allyl- $N-(p-$ toluenesulfonyl)amino]-4'-fluorophenyl)prop-2-enoate (5e) (0.946 g, 
$79 \%$ ) as a white solid. Mp $111-113{ }^{\circ} \mathrm{C} ; R_{f}=0.38$ (diethyl ether/ petroleum ether $=1: 1)$; IR (neat) 2951, 1712, 1602, 1497, 1353, 1256, 1164, 908, $730 \mathrm{~cm}^{-1}$; ${ }^{1} \mathrm{H}$ NMR $\left(400 \mathrm{MHz}, \mathrm{CDCl}_{3}\right) \delta 2.43(\mathrm{~s}, 3 \mathrm{H})$, $3.78(\mathrm{~s}, 3 \mathrm{H}), 4.02(\mathrm{br} \mathrm{s}, 1 \mathrm{H}), 4.21(\mathrm{br} \mathrm{s}, 1 \mathrm{H}), 4.95-5.05(\mathrm{~m}, 2 \mathrm{H}), 5.72$ (ddt, $J=16.9,10.1,6.8 \mathrm{~Hz}, 1 \mathrm{H}), 6.27(\mathrm{~d}, J=16.1 \mathrm{~Hz}, 1 \mathrm{H}), 6.57(\mathrm{dd}$, $\left.{ }^{3} J_{H F}=9.2, J=2.8 \mathrm{~Hz}, 1 \mathrm{H}\right), 7.06(\mathrm{td}, J=8.8,2.8 \mathrm{~Hz}, 1 \mathrm{H}), 7.29(\mathrm{~d}, J=$ $8.2 \mathrm{~Hz}, 2 \mathrm{H}), 7.58(\mathrm{~d}, J=8.2 \mathrm{~Hz}, 2 \mathrm{H}), 7.62\left(\mathrm{dd}, J=8.8,{ }^{4} J_{\mathrm{HF}}=6.2 \mathrm{~Hz}\right.$, $1 \mathrm{H}), 7.78(\mathrm{~d}, J=16.1 \mathrm{~Hz}, 1 \mathrm{H}) ;{ }^{13} \mathrm{C}$ NMR $\left(101 \mathrm{MHz}, \mathrm{CDCl}_{3}\right) \delta 21.6$ $\left(\mathrm{CH}_{3}\right), 51.7\left(\mathrm{CH}_{3}\right), 54.9\left(\mathrm{CH}_{2}\right), 116.4\left(\mathrm{~d},{ }^{2} J_{\mathrm{CF}}=21.6 \mathrm{~Hz}, \mathrm{CH}\right), 117.0$ $\left(\mathrm{d},{ }^{2} J_{\mathrm{CF}}=21.9 \mathrm{~Hz}, \mathrm{CH}\right), 119.5(\mathrm{CH}), 120.2\left(\mathrm{CH}_{2}\right), 128.0(2 \times \mathrm{CH})$, $128.6\left(\mathrm{~d},{ }^{3} J_{\mathrm{CF}}=9.4 \mathrm{~Hz}, \mathrm{CH}\right), 129.7(2 \times \mathrm{CH}), 131.7(\mathrm{CH}), 132.1(\mathrm{~d}$, $\left.{ }^{4} J_{C F}=3.7 \mathrm{~Hz}, \mathrm{C}\right), 135.1(\mathrm{C}), 139.3(\mathrm{CH}), 139.8\left(\mathrm{~d},{ }^{3} J_{\mathrm{CF}}=9.2 \mathrm{~Hz}, \mathrm{C}\right)$, 144.2 (C), 163.1 (d, $\left.{ }^{1} J_{C F}=253.1 \mathrm{~Hz}, \mathrm{C}\right), 166.7(\mathrm{C}) ; \mathrm{MS}(\mathrm{ESI}) \mathrm{m} / z$ $412\left(\mathrm{MNa}^{+}, 100\right)$; HRMS (ESI) calcd for $\mathrm{C}_{20} \mathrm{H}_{20} \mathrm{FNNaO}_{4} \mathrm{~S}\left(\mathrm{MNa}^{+}\right)$, 412.0989; found, 412.0970.

Methyl (2E)-3-(2'-[N-Allyl- $N$-( $p$-toluenesulfonyl)amino]-5'chlorophenyl)prop-2-enoate (5f). The reaction was carried out as described for the synthesis of methyl (2E)-3- $\left(2^{\prime}-[N-\right.$ allyl- $N-(p-$ toluenesulfonyl)amino]phenyl)prop-2-enoate (5a) using methyl $(2 E)$ 3-(5'-chloro-2' $-[N$-( $p$-toluenesulfonyl)amino $]$ phenyl) prop-2-enoate $(0.600 \mathrm{~g}, 1.60 \mathrm{mmol})$. Purification by column chromatography (ethyl acetate/petroleum ether $=1: 5)$ gave methyl $(2 E)-3-\left(2^{\prime}-[N\right.$-allyl $-N-(p-$ toluenesulfonyl)amino]-5' -chlorophenyl)prop-2-enoate (5f) (0.664 g, $100 \%$ ) as a yellow solid. Mp $104-106^{\circ} \mathrm{C} ; R_{f}=0.58$ (petroleum ether/ ethyl acetate $=2: 1$ ); IR (neat) 2951, 1720, 1610, 1353, 1164, 908, 730 $\mathrm{cm}^{-1} ;{ }^{1} \mathrm{H}$ NMR (400 MHz, $\left.\mathrm{CDCl}_{3}\right) \delta 2.44(\mathrm{~s}, 3 \mathrm{H}), 3.80(\mathrm{~s}, 3 \mathrm{H}), 3.99$ (br s, $1 \mathrm{H}), 4.26(\mathrm{br} \mathrm{s}, 1 \mathrm{H}), 4.97(\mathrm{dd}, J=17.0,1.1 \mathrm{~Hz}, 1 \mathrm{H}), 5.03(\mathrm{dd}, J$ $=10.1,1.1 \mathrm{~Hz}, 1 \mathrm{H}), 5.73(\mathrm{ddt}, J=17.0,10.1,6.8 \mathrm{~Hz}, 1 \mathrm{H}), 6.31(\mathrm{~d}, J=$ $16.1 \mathrm{~Hz}, 1 \mathrm{H}), 6.78(\mathrm{~d}, J=8.6 \mathrm{~Hz}, 1 \mathrm{H}), 7.24(\mathrm{dd}, J=8.6,2.4 \mathrm{~Hz}, 1 \mathrm{H})$, $7.29(\mathrm{~d}, J=8.2 \mathrm{~Hz}, 2 \mathrm{H}), 7.57(\mathrm{~d}, J=8.2 \mathrm{~Hz}, 2 \mathrm{H}), 7.60(\mathrm{~d}, J=2.4 \mathrm{~Hz}$, $1 \mathrm{H}), 7.75(\mathrm{~d}, J=16.1 \mathrm{~Hz}, 1 \mathrm{H}) ;{ }^{13} \mathrm{C}$ NMR $\left(101 \mathrm{MHz}, \mathrm{CDCl}_{3}\right) \delta 21.6$ $\left(\mathrm{CH}_{3}\right), 51.8\left(\mathrm{CH}_{3}\right), 54.9\left(\mathrm{CH}_{2}\right), 120.2\left(\mathrm{CH}_{2}\right), 121.0(\mathrm{CH}), 127.1$ $(\mathrm{CH}), 128.0(2 \times \mathrm{CH}), 129.7(2 \times \mathrm{CH}), 130.3(\mathrm{CH}), 131.2(\mathrm{CH})$, $131.8(\mathrm{CH}), 134.7(\mathrm{C}), 135.3(\mathrm{C}), 136.7(\mathrm{C}), 137.4(\mathrm{C}), 139.0(\mathrm{CH})$, 144.1 (C), 166.5 (C); MS (ESI) $m / z 428\left(\mathrm{MNa}^{+}, 100\right)$; HRMS (ESI) calcd for $\mathrm{C}_{20} \mathrm{H}_{20}{ }^{35} \mathrm{ClNNaO} \mathrm{S}_{4}\left(\mathrm{MNa}^{+}\right)$, 428.0694; found, 428.0673 .

(2E)-3-(2'-[N-Allyl- $N$-(p-toluenesulfonyl)amino]phenyl)prop2-en-1-ol (6a). Diisobutylaluminum hydride $(4.1 \mathrm{~mL}, 4.1 \mathrm{mmol}, 1 \mathrm{M}$ in hexane) was added dropwise with stirring to a solution of methyl (2E)-3-(2'-[N-allyl- $N$ - ( $p$-toluenesulfonyl) amino $]$ phenyl)prop-2enoate (5a) $(0.690 \mathrm{~g}, 1.86 \mathrm{mmol})$ in dichloromethane $(19 \mathrm{~mL})$ at -78 ${ }^{\circ} \mathrm{C}$. The solution was stirred at $-78{ }^{\circ} \mathrm{C}$ for $2 \mathrm{~h}$ and then allowed to warm to room temperature over $16 \mathrm{~h}$. The reaction was quenched with $10 \%$ aqueous potassium sodium tartrate solution $(5 \mathrm{~mL})$, extracted with diethyl ether $(2 \times 10 \mathrm{~mL})$, washed with water $(20 \mathrm{~mL})$, brine $(20$ $\mathrm{mL})$, dried $\left(\mathrm{MgSO}_{4}\right)$, filtered, and concentrated in vacuo. Purification by column chromatography (ethyl acetate/petroleum ether $=1: 2$ ) gave (2E)-3-(2'-[N-allyl- $N$-( $p$-toluenesulfonyl) amino $]$ phenyl $)$ prop-2en-1-ol (6a) $(0.611 \mathrm{~g}, 96 \%)$ as a colorless oil. $R_{f}=0.13$ (diethyl ether/petroleum ether =1:1); IR (neat) 3491, 2924, 1597, 1341, 1161, $726 \mathrm{~cm}^{-1} ;{ }^{1} \mathrm{H}$ NMR (400 MHz, $\left.\mathrm{CDCl}_{3}\right) \delta 1.97$ (br s, $\left.1 \mathrm{H}\right), 2.43(\mathrm{~s}$, $3 \mathrm{H}), 4.00(\mathrm{br} \mathrm{s}, 1 \mathrm{H}), 4.18-4.29(\mathrm{~m}, 3 \mathrm{H}), 4.93-5.01(\mathrm{~m}, 2 \mathrm{H}), 5.72$ $(\mathrm{ddt}, J=16.9,10.2,6.7 \mathrm{~Hz}, 1 \mathrm{H}), 6.33(\mathrm{dt}, J=16.0,5.4 \mathrm{~Hz}, 1 \mathrm{H}), 6.68$ $(\mathrm{dd}, J=7.8,1.3 \mathrm{~Hz}, 1 \mathrm{H}), 6.83(\mathrm{~d}, J=16.0 \mathrm{~Hz}, 1 \mathrm{H}), 7.12(\mathrm{td}, J=7.8$, $1.3 \mathrm{~Hz}, 1 \mathrm{H}), 7.23-7.30(\mathrm{~m}, 3 \mathrm{H}), 7.55-7.61(\mathrm{~m}, 3 \mathrm{H}) ;{ }^{13} \mathrm{C}$ NMR $(101$ $\left.\mathrm{MHz}, \mathrm{CDCl}_{3}\right) \delta 21.5\left(\mathrm{CH}_{3}\right), 54.8\left(\mathrm{CH}_{2}\right), 63.8\left(\mathrm{CH}_{2}\right), 119.4\left(\mathrm{CH}_{2}\right)$, $126.5(\mathrm{CH}), 126.7(\mathrm{CH}), 127.8(\mathrm{CH}), 127.9(2 \times \mathrm{CH}), 128.6(\mathrm{CH})$, $129.4(\mathrm{CH}), 129.5(2 \times \mathrm{CH}), 130.8(\mathrm{CH}), 132.4(\mathrm{CH}), 136.1(\mathrm{C})$, 136.6 (C), 137.8 (C), 143.6 (C); MS (ESI) $m / z 366\left(\mathrm{MNa}^{+}, 100\right)$; HMRS (ESI) calcd for $\mathrm{C}_{19} \mathrm{H}_{21} \mathrm{NNaO}_{3} \mathrm{~S}\left(\mathrm{MNa}^{+}\right), 366.1134$; found, 366.1119.

(2E)-3-(2'-[N-Allyl- $N$-( $p$-toluenesulfonyl)amino]-5'-methylphenyl)prop-2-en-1-ol (6b). The reaction was carried out as described for the synthesis of $(2 E)-3-\left(2^{\prime}-[N\right.$-allyl- $N-(p$-toluenesulfonyl)amino]phenyl)prop-2-en-1-ol (6a) using methyl (2E)-3-(2'[ $N$-allyl- $N$-( $p$-toluenesulfonyl)amino]-5'-methylphenyl)prop-2-enoate (5b) $(1.50 \mathrm{~g}, 3.89 \mathrm{mmol})$. Purification by column chromatography (ethyl acetate/petroleum ether $=1: 2)$ gave $(2 E)-3-\left(2^{\prime}-[N\right.$-allyl $-N-(p-$ toluenesulfonyl)amino]-5' -methylphenyl)prop-2-en-1-ol (6b) (1.37 g, $98 \%$ ) as a colorless oil. $R_{f}=0.10$ (diethyl ether/petroleum ether $=$
1:1); IR (neat) 3510, 2921, 1598, 1491, 1340, 1159, 859, $734 \mathrm{~cm}^{-1}$; ${ }^{1} \mathrm{H}$ NMR (400 MHz, $\left.\mathrm{CDCl}_{3}\right) \delta 2.15($ br s, $1 \mathrm{H}), 2.31(\mathrm{~s}, 3 \mathrm{H}), 2.42(\mathrm{~s}$, $3 \mathrm{H}), 3.96($ br s, $1 \mathrm{H}), 4.19-4.28(\mathrm{~m}, 3 \mathrm{H}), 4.93-5.01(\mathrm{~m}, 2 \mathrm{H}), 5.72$ $(\mathrm{ddt}, J=16.9,10.2,6.7 \mathrm{~Hz}, 1 \mathrm{H}), 6.31(\mathrm{dt}, J=16.0,5.7 \mathrm{~Hz}, 1 \mathrm{H}), 6.56$ $(\mathrm{d}, J=8.1 \mathrm{~Hz}, 1 \mathrm{H}), 6.79(\mathrm{~d}, J=16.0 \mathrm{~Hz}, 1 \mathrm{H}), 6.92(\mathrm{dd}, J=8.1,1.3$ $\mathrm{Hz}, 1 \mathrm{H}), 7.27(\mathrm{~d}, J=8.2 \mathrm{~Hz}, 2 \mathrm{H}), 7.40(\mathrm{~d}, J=1.3 \mathrm{~Hz}, 1 \mathrm{H}), 7.57(\mathrm{~d}, J$ $=8.2 \mathrm{~Hz}, 2 \mathrm{H}) ;{ }^{13} \mathrm{C} \mathrm{NMR}\left(101 \mathrm{MHz}, \mathrm{CDCl}_{3}\right) \delta 21.2\left(\mathrm{CH}_{3}\right), 21.5$ $\left(\mathrm{CH}_{3}\right), 54.8\left(\mathrm{CH}_{2}\right), 63.8\left(\mathrm{CH}_{2}\right), 119.2\left(\mathrm{CH}_{2}\right), 126.7(\mathrm{CH}), 127.0$ $(\mathrm{CH}), 127.9(2 \times \mathrm{CH}), 128.7(\mathrm{CH}), 129.1(\mathrm{CH}), 129.5(2 \times \mathrm{CH})$, 130.5 (CH), $132.5(\mathrm{CH}), 134.1$ (C), 136.2 (C), 137.3 (C), $138.4(\mathrm{C})$, 143.5 (C); MS (ESI) $\mathrm{m} / z 380\left(\mathrm{MNa}^{+}, 100\right)$; HRMS (ESI) calcd for $\mathrm{C}_{20} \mathrm{H}_{23} \mathrm{NNaO}_{3} \mathrm{~S}\left(\mathrm{MNa}^{+}\right)$, 380.1291; found, 380.1279.

(2E)-3-(2'-[N-Allyl- $N$-( $p$-toluenesulfonyl)amino]-5'-methoxyphenyl)prop-2-en-1-ol (6c). The reaction was carried out as described for the synthesis of (2E)-3-(2'-[N-allyl- $N-(p$-toluenesulfonyl)amino]phenyl)prop-2-en-1-ol (6a) using methyl (2E)-3-(2'[N-allyl- $N$-( $p$-toluenesulfonyl)amino]-5' -methoxyphenyl)prop-2enoate $(5 \mathrm{c})(0.140 \mathrm{~g}, 0.350 \mathrm{mmol})$. Purification by column chromatography (ethyl acetate/petroleum ether $=1: 2$ ) gave $(2 E)-3$ $\left(2^{\prime}\right.$ - $[N$-allyl- $N$-( $p$-toluenesulfonyl)amino $]-5^{\prime}$-methoxyphenyl)prop-2en-1-ol $(6 \mathrm{c})(0.104 \mathrm{~g}, 80 \%)$ as a colorless oil. $R_{f}=0.18$ (petroleum ether/ethyl acetate $=2: 1$ ); IR (neat) 3523, 2944, 1601, 1495, 1345, $1161,752 \mathrm{~cm}^{-1}$; ${ }^{1} \mathrm{H}$ NMR $\left(400 \mathrm{MHz}, \mathrm{CDCl}_{3}\right) \delta 2.07(\mathrm{t}, J=5.4 \mathrm{~Hz}$, $1 \mathrm{H}), 2.42(\mathrm{~s}, 3 \mathrm{H}), 3.79(\mathrm{~s}, 3 \mathrm{H}), 3.91-3.98(\mathrm{~m}, 1 \mathrm{H}), 4.18-4.28(\mathrm{~m}$, $3 \mathrm{H}), 4.93-5.01(\mathrm{~m}, 2 \mathrm{H}), 5.72(\mathrm{ddt}, J=16.9,10.2,6.7 \mathrm{~Hz}, 1 \mathrm{H}), 6.30$ $(\mathrm{dt}, J=16.0,5.4 \mathrm{~Hz}, 1 \mathrm{H}), 6.58(\mathrm{~d}, J=8.8 \mathrm{~Hz}, 1 \mathrm{H}), 6.65(\mathrm{dd}, J=8.8$, $2.9 \mathrm{~Hz}, 1 \mathrm{H}), 6.77(\mathrm{dt}, J=16.0,1.5 \mathrm{~Hz}, 1 \mathrm{H}), 7.07(\mathrm{~d}, J=2.9 \mathrm{~Hz}, 1 \mathrm{H})$, $7.27(\mathrm{~d}, J=8.2 \mathrm{~Hz}, 2 \mathrm{H}), 7.57(\mathrm{~d}, J=8.2 \mathrm{~Hz}, 2 \mathrm{H}) ;{ }^{13} \mathrm{C}$ NMR $(101$ $\left.\mathrm{MHz}, \mathrm{CDCl}_{3}\right) \delta 21.5\left(\mathrm{CH}_{3}\right), 54.9\left(\mathrm{CH}_{2}\right), 55.4\left(\mathrm{CH}_{3}\right), 63.7\left(\mathrm{CH}_{2}\right)$, $110.7(\mathrm{CH}), 113.9(\mathrm{CH}), 119.3\left(\mathrm{CH}_{2}\right), 126.6(\mathrm{CH}), 127.9(2 \times \mathrm{CH})$, $129.4(\mathrm{C}), 129.7(2 \times \mathrm{CH}), 130.4(\mathrm{CH}), 130.9(\mathrm{CH}), 132.5(\mathrm{CH})$, 136.2 (C), 138.8 (C), 143.5 (C), 159.2 (C); MS (ESI) $m / z 396$ $\left(\mathrm{MNa}^{+}, 100\right)$; HRMS (ESI) calcd for $\mathrm{C}_{20} \mathrm{H}_{23} \mathrm{NNaO}_{4} \mathrm{~S}\left(\mathrm{MNa}^{+}\right)$, 396.1240; found, 396.1223.

(2E)-3-(2' - [N-Allyl- $N$-(p-toluenesulfonyl) amino $]-5^{\prime}$ fluorophenyl)prop-2-en-1-ol (6d). The reaction was carried out as described for the synthesis of $(2 E)-3-\left(2^{\prime}-[N\right.$-allyl- $N-(p$-toluenesulfonyl)amino]phenyl)prop-2-en-1-ol (6a) using methyl (2E)-3-(2'[ $N$-allyl- $N$-( $p$-toluenesulfonyl)amino]-5'-fluorophenyl)prop-2-enoate (5d) $(3.30 \mathrm{~g}, 8.50 \mathrm{mmol})$. Purification by column chromatography (ethyl acetate/petroleum ether $=1: 3)$ gave $(2 E)-3-\left(2^{\prime}-[N\right.$-allyl- $N-(p-$ toluenesulfonyl)amino]-5'-fluorophenyl)prop-2-en-1-ol (6d) (2.99 g, $98 \%$ ) as a colorless oil. $R_{f}=0.10$ (diethyl ether/petroleum ether $=$ $1: 1$ ); IR (neat) $3507,2920,1600,1488,1345,1161,752 \mathrm{~cm}^{-1} ;{ }^{1} \mathrm{H}$ $\operatorname{NMR}\left(400 \mathrm{MHz}, \mathrm{CDCl}_{3}\right) \delta 2.35(\mathrm{t}, J=5.6 \mathrm{~Hz}, 1 \mathrm{H}), 2.42(\mathrm{~s}, 3 \mathrm{H}), 3.95$ $(\mathrm{dd}, J=13.4,6.8,1 \mathrm{H}), 4.17-4.28(\mathrm{~m}, 3 \mathrm{H}), 4.91-5.01(\mathrm{~m}, 2 \mathrm{H}), 5.70$ (ddt, $J=16.9,10.1,6.8 \mathrm{~Hz}, 1 \mathrm{H}), 6.30(\mathrm{dt}, J=16.0,5.5 \mathrm{~Hz}, 1 \mathrm{H}), 6.65$ $\left(\mathrm{dd}, J=8.8,{ }^{4} J_{H F}=5.4 \mathrm{~Hz}, 1 \mathrm{H}\right), 6.73-6.83(\mathrm{~m}, 2 \mathrm{H}), 7.24\left(\mathrm{dd},{ }^{3} J_{\mathrm{HF}}=\right.$ $10.0, J=2.9 \mathrm{~Hz}, 1 \mathrm{H}), 7.27(\mathrm{~d}, J=8.3 \mathrm{~Hz}, 2 \mathrm{H}), 7.56(\mathrm{~d}, J=8.3 \mathrm{~Hz}$, $2 \mathrm{H}) ;{ }^{13} \mathrm{C}$ NMR $\left(101 \mathrm{MHz}, \mathrm{CDCl}_{3}\right) \delta 21.5\left(\mathrm{CH}_{3}\right), 54.9\left(\mathrm{CH}_{2}\right), 63.4$ $\left(\mathrm{CH}_{2}\right), 112.8\left(\mathrm{~d},{ }^{2} J_{\mathrm{CF}}=23.3 \mathrm{~Hz}, \mathrm{CH}\right), 114.7\left(\mathrm{~d},{ }^{2} J_{\mathrm{CF}}=23.1 \mathrm{~Hz}, \mathrm{CH}\right)$, $119.7\left(\mathrm{CH}_{2}\right), 125.4\left(\mathrm{~d},{ }^{4} J_{\mathrm{CF}}=1.7 \mathrm{~Hz}, \mathrm{CH}\right), 127.9(2 \times \mathrm{CH}), 129.6(2$ $\times \mathrm{CH}), 131.2\left(\mathrm{~d},{ }^{3} J_{\mathrm{CF}}=9.1 \mathrm{~Hz}, \mathrm{CH}\right), 132.1(\mathrm{CH}), 132.3(\mathrm{CH}), 132.4$ $\left(\mathrm{d},{ }^{4} J_{\mathrm{CF}}=2.8 \mathrm{~Hz}, \mathrm{C}\right), 135.9(\mathrm{C}), 140.2\left(\mathrm{~d},{ }^{3} J_{\mathrm{CF}}=8.6 \mathrm{~Hz}, \mathrm{C}\right), 143.8$ (C), $162.2\left(\mathrm{~d},{ }^{1} J_{\mathrm{CF}}=247.9 \mathrm{~Hz}, \mathrm{C}\right)$; MS (ESI) $\mathrm{m} / z 384\left(\mathrm{MNa}^{+}, 100\right)$; HRMS (ESI) calcd for $\mathrm{C}_{19} \mathrm{H}_{20} \mathrm{FNNaO}_{3} \mathrm{~S}\left(\mathrm{MNa}^{+}\right)$, 384.1040; found, 384.1023 .

(2E)-3-(2'-[N-Allyl- $N$-( $p$-toluenesulfonyl)amino]-4' -fluorophenyl)prop-2-en-1-ol (6e). The reaction was carried out as described for the synthesis of (2E)-3-(2'-[N-allyl- $N-(p$-toluenesulfonyl)amino]phenyl)prop-2-en-1-ol (6a) using methyl (2E)-3-(2'[ $N$-allyl- $N$-( $p$-toluenesulfonyl)amino]-4'-fluorophenyl)prop-2-enoate (5e) $(0.790 \mathrm{~g}, 2.00 \mathrm{mmol})$. Purification by column chromatography (ethyl acetate/petroleum ether, 1:3) gave $(2 E)-3-\left(2^{\prime}-[N\right.$-allyl- $N-(p$ toluenesulfonyl)amino]-4'-fluorophenyl)prop-2-en-1-ol (6e) (0.728 g, $99 \%)$ as a colorless oil. $R_{f}=0.08$ (diethyl ether/petroleum ether $=$ $1: 1$ ); IR (neat) $3507,2923,1600,1495,1347,1161,908,727 \mathrm{~cm}^{-1}$; ${ }^{1} \mathrm{H}$ NMR $\left(400 \mathrm{MHz}, \mathrm{CDCl}_{3}\right) \delta 2.18(\mathrm{t}, J=5.6 \mathrm{~Hz}, 1 \mathrm{H}), 2.43(\mathrm{~s}, 3 \mathrm{H})$, $3.96($ br s, $1 \mathrm{H}), 4.09-4.27(\mathrm{~m}, 3 \mathrm{H}), 4.94-5.03(\mathrm{~m}, 2 \mathrm{H}), 5.69$ (ddt, $J=$ $16.9,10.1,6.7 \mathrm{~Hz}, 1 \mathrm{H}), 6.25(\mathrm{dt}, J=16.0,5.3 \mathrm{~Hz}, 1 \mathrm{H}), 6.41\left(\mathrm{dd},{ }^{3} J_{\mathrm{HF}}\right.$ 
$=9.3, J=2.6 \mathrm{~Hz}, 1 \mathrm{H}), 6.75(\mathrm{~d}, J=16.0 \mathrm{~Hz}, 1 \mathrm{H}), 6.99(\mathrm{td}, J=8.6,2.6$ $\mathrm{Hz}, 1 \mathrm{H}), 7.29(\mathrm{~d}, J=8.2 \mathrm{~Hz}, 2 \mathrm{H}), 7.55\left(\mathrm{dd}, J=8.6,{ }^{4} J_{\mathrm{HF}}=6.3 \mathrm{~Hz}\right.$, $1 \mathrm{H}), 7.58(\mathrm{~d}, J=8.2 \mathrm{~Hz}, 2 \mathrm{H}) ;{ }^{13} \mathrm{C}$ NMR $\left(101 \mathrm{MHz}, \mathrm{CDCl}_{3}\right) \delta 21.6$ $\left(\mathrm{CH}_{3}\right), 54.7\left(\mathrm{CH}_{2}\right), 63.6\left(\mathrm{CH}_{2}\right), 116.0\left(\mathrm{~d},{ }^{2} J_{\mathrm{CF}}=21.2 \mathrm{~Hz}, \mathrm{CH}\right), 116.2$ $\left(\mathrm{d},{ }^{2} J_{\mathrm{CF}}=21.2 \mathrm{~Hz}, \mathrm{CH}\right), 119.8\left(\mathrm{CH}_{2}\right), 125.7(\mathrm{CH}), 127.7\left(\mathrm{~d},{ }^{3} J_{\mathrm{CF}}=\right.$ $8.9 \mathrm{~Hz}, \mathrm{CH}), 127.9(2 \times \mathrm{CH}), 129.7(2 \times \mathrm{CH}), 130.7\left(\mathrm{~d},{ }^{5} J_{\mathrm{CF}}=1.8\right.$ $\mathrm{Hz}, \mathrm{CH}), 131.9(\mathrm{CH}), 134.3\left(\mathrm{~d},{ }^{4} J_{\mathrm{CF}}=3.7 \mathrm{~Hz}, \mathrm{C}\right), 135.7(\mathrm{C}), 137.7$ $\left(\mathrm{d},{ }^{3} J_{\mathrm{CF}}=8.8 \mathrm{~Hz}, \mathrm{C}\right), 144.0(\mathrm{C}), 161.5\left(\mathrm{~d},{ }^{1} J_{\mathrm{CF}}=248.9 \mathrm{~Hz}, \mathrm{C}\right)$; MS (ESI) $m / z 384\left(\mathrm{MNa}^{+}, 100\right)$; HRMS (ESI) calcd for $\mathrm{C}_{19} \mathrm{H}_{20} \mathrm{FNNaO}_{3} \mathrm{~S}$ $\left(\mathrm{MNa}^{+}\right)$, 384.1040; found, 384.1023.

(2E)-3-(2'-[N-Allyl- $N$-(p-toluenesulfonyl)amino]-5' -chlorophenyl)prop-2-en-1-ol (6f). The reaction was carried out as described for the synthesis of (2E)-3-(2'-[N-allyl- $N$ - $(p$-toluenesulfonyl)amino]phenyl)prop-2-en-1-ol (6a) using methyl (2E)-3-(2'[ $N$-allyl- $N$-( $p$-toluenesulfonyl)amino]- $5^{\prime}$-chlorophenyl)prop-2-enoate (5f) $(0.660 \mathrm{~g}, 1.60 \mathrm{mmol})$. Purification by column chromatography (ethyl acetate/petroleum ether $=1: 3)$ gave $(2 E)-3-\left(2^{\prime}-[N\right.$-allyl- $N$ - $(p-$ toluenesulfonyl)amino]-5' -chlorophenyl)prop-2-en-1-ol (6f) (0.566 g, $92 \%$ ) as a colorless oil. $R_{f}=0.28$ (petroleum ether/ethyl acetate $=$ 2:1); IR (neat) $3505,2923,1597,1478,1343,1161,907,727 \mathrm{~cm}^{-1}$; ${ }^{1} \mathrm{H}$ NMR $\left(400 \mathrm{MHz}, \mathrm{CDCl}_{3}\right) \delta 2.23(\mathrm{br} \mathrm{s}, 1 \mathrm{H}), 2.43(\mathrm{~s}, 3 \mathrm{H}), 3.94(\mathrm{br}$ $\mathrm{s}, 1 \mathrm{H}), 4.17-4.29(\mathrm{~m}, 3 \mathrm{H}), 4.92-5.02(\mathrm{~m}, 2 \mathrm{H}), 5.69(\mathrm{ddt}, J=17.0$, $10.1,6.8 \mathrm{~Hz}, 1 \mathrm{H}), 6.32(\mathrm{dt}, J=16.0,5.1 \mathrm{~Hz}, 1 \mathrm{H}), 6.60(\mathrm{~d}, J=8.6 \mathrm{~Hz}$, $1 \mathrm{H}), 6.75(\mathrm{dt}, J=16.0,1.5 \mathrm{~Hz}, 1 \mathrm{H}), 7.07(\mathrm{dd}, J=8.6,2.4 \mathrm{~Hz}, 1 \mathrm{H})$, $7.28(\mathrm{~d}, J=8.2 \mathrm{~Hz}, 2 \mathrm{H}), 7.54-7.59(\mathrm{~m}, 3 \mathrm{H}) ;{ }^{13} \mathrm{C} \mathrm{NMR}(101 \mathrm{MHz}$, $\left.\mathrm{CDCl}_{3}\right) \delta 21.6\left(\mathrm{CH}_{3}\right), 54.8\left(\mathrm{CH}_{2}\right), 63.4\left(\mathrm{CH}_{2}\right), 119.8\left(\mathrm{CH}_{2}\right), 125.2$ $(\mathrm{CH}), 126.5(\mathrm{CH}), 127.8(\mathrm{CH}), 127.9(2 \times \mathrm{CH}), 129.7(2 \times \mathrm{CH})$, $130.7(\mathrm{CH}), 132.0(\mathrm{CH}), 132.4(\mathrm{CH}), 134.5(\mathrm{C}), 135.0(\mathrm{C}), 135.8$ (C), 139.6 (C), 143.9 (C); MS (ESI) $m / z 400\left(\mathrm{MNa}^{+}, 100\right)$; HRMS (ESI) calcd for $\mathrm{C}_{19} \mathrm{H}_{20}{ }^{35} \mathrm{ClNNaO}_{3} \mathrm{~S}\left(\mathrm{MNa}^{+}\right), 400.0745$; found, 400.0729 .

5-Nitro-2-[N-(p-toluenesulfonyl)amino]benzaldehyde (8). $p$ Toluenesulfonamide $(0.148 \mathrm{~g}, 0.865 \mathrm{mmol})$ was added to a solution of 2-chloro-5-nitrobenzaldehyde (7) (0.0800 g, $0.432 \mathrm{mmol})$, and potassium carbonate $(0.107 \mathrm{~g}, 0.780 \mathrm{mmol})$ in $N, N^{\prime}$-dimethylformamide $(2 \mathrm{~mL})$ and heated to $90{ }^{\circ} \mathrm{C}$ for $2 \mathrm{~h}$. The reaction mixture was cooled to room temperature, diluted with water $(2 \mathrm{~mL})$, and extracted with ethyl acetate $(10 \mathrm{~mL})$. The organic layer was washed with $1 \mathrm{M}$ hydrochloric acid solution $(3 \times 2 \mathrm{~mL})$ and brine $(2$ $\mathrm{mL})$, dried $\left(\mathrm{MgSO}_{4}\right)$, filtered, and concentrated in vacuo. Purification by column chromatography (ethyl acetate/petroleum ether $=1: 5$ ) gave 5-nitro-2-[ $\mathrm{N}$-( $p$-toluenesulfonyl)amino $]$ benzaldehyde $(8)(0.122$ $\mathrm{g}, 86 \%)$ as a white solid. $\mathrm{Mp} 172-174{ }^{\circ} \mathrm{C} ; R_{f}=0.38$ (petroleum ether/ ethyl acetate $=2: 1$ ); IR (neat) $3164,1673,1586,1345,1215,1164$, $749 \mathrm{~cm}^{-1} ;{ }^{1} \mathrm{H}$ NMR $\left(400 \mathrm{MHz}, \mathrm{CDCl}_{3}\right) \delta 2.40(\mathrm{~s}, 3 \mathrm{H}), 7.31(\mathrm{~d}, J=$ $8.2 \mathrm{~Hz}, 2 \mathrm{H}), 7.81(\mathrm{~d}, J=9.3 \mathrm{~Hz}, 1 \mathrm{H}), 7.83(\mathrm{~d}, J=8.2 \mathrm{~Hz}, 2 \mathrm{H}), 8.34$ $(\mathrm{dd}, J=9.3,2.6 \mathrm{~Hz}, 1 \mathrm{H}), 8.54(\mathrm{~d}, J=2.6 \mathrm{~Hz}, 1 \mathrm{H}), 9.94(\mathrm{~d}, J=0.6 \mathrm{~Hz}$, $1 \mathrm{H}), 11.19($ br s, $1 \mathrm{H}) ;{ }^{13} \mathrm{C} \mathrm{NMR}\left(101 \mathrm{MHz}, \mathrm{CDCl}_{3}\right) \delta 21.6\left(\mathrm{CH}_{3}\right)$, $117.3(\mathrm{CH}), 120.5(\mathrm{C}), 127.4(2 \times \mathrm{CH}), 130.2(2 \times \mathrm{CH}), 130.5$ (CH), 131.5 (CH), 135.6 (C), 142.1 (C), 145.0 (C), 145.3 (C), 193.5 $(\mathrm{CH})$; MS (ESI) $\mathrm{m} / z 343\left(\mathrm{MNa}^{+}, 100\right)$; HRMS (ESI) calcd for $\mathrm{C}_{14} \mathrm{H}_{12} \mathrm{~N}_{2} \mathrm{NaO}_{5} \mathrm{~S}\left(\mathrm{MNa}^{+}\right)$, 343.0359; found, 343.0350.

Ethyl (2E)-3-(5'-Nitro-2'-[N-(p-toluenesulfonyl)amino]phenyl)prop-2-enoate. Lithium bromide $(0.043 \mathrm{~g}, 0.50 \mathrm{mmol})$ was added to a solution of triethyl phosphonoacetate $(0.085 \mathrm{~mL}, 0.43$ $\mathrm{mmol})$ and 1,8-diazabicyclo[5.4.0] undec-7-ene $(0.064 \mathrm{~mL}, 0.43$ $\mathrm{mmol})$ in acetonitrile $(2 \mathrm{~mL})$ and stirred at room temperature for 0.5 h. 5-Nitro-2-[N-( $p$-toluenesulfonyl)amino]benzaldehyde (8) $(0.040 \mathrm{~g}, 0.13 \mathrm{mmol})$ was added, and the solution was stirred at room temperature for $3 \mathrm{~h}$. The reaction was quenched with $10 \%$ aqueous potassium sodium tartrate solution $(2 \mathrm{~mL})$, concentrated to half volume in vacuo, and extracted with diethyl ether $(3 \times 5 \mathrm{~mL})$. The combined organic layers were washed with water $(2 \mathrm{~mL})$, brine $(2$ $\mathrm{mL})$, dried $\left(\mathrm{MgSO}_{4}\right)$, filtered, and concentrated in vacuo. Purification by column chromatography (ethyl acetate/petroleum ether $=1: 3$ ) gave ethyl (2E)-3-( $5^{\prime}$-nitro- $2^{\prime}$-[N-( $p$-toluenesulfonyl)amino $]$ phenyl)prop-2-enoate $(0.048 \mathrm{~g}, 99 \%)$ as a white solid. $\mathrm{Mp} 158-160{ }^{\circ} \mathrm{C} ; R_{f}=$ 0.28 (petroleum ether/ethyl acetate $=2: 1$ ); IR (neat) 3255, 2980, 1700, 1640, 1527, 1344, 1166, 908, $757 \mathrm{~cm}^{-1}$; ${ }^{1} \mathrm{H}$ NMR $(400 \mathrm{MHz}$, $\left.\mathrm{CDCl}_{3}\right) \delta 1.34(\mathrm{t}, J=7.1 \mathrm{~Hz}, 3 \mathrm{H}), 2.38(\mathrm{~s}, 3 \mathrm{H}), 4.27(\mathrm{q}, J=7.1 \mathrm{~Hz}$,
$2 \mathrm{H}), 6.35(\mathrm{~d}, J=15.7 \mathrm{~Hz}, 1 \mathrm{H}), 7.26(\mathrm{~d}, J=8.2 \mathrm{~Hz}, 2 \mathrm{H}), 7.63(\mathrm{~d}, J=$ $15.7 \mathrm{~Hz}, 1 \mathrm{H}), 7.65-7.72(\mathrm{~m}, 4 \mathrm{H}), 8.16(\mathrm{dd}, J=9.0,2.6 \mathrm{~Hz}, 1 \mathrm{H}), 8.28$ $(\mathrm{d}, J=2.6 \mathrm{~Hz}, 1 \mathrm{H}) ;{ }^{13} \mathrm{C} \mathrm{NMR}\left(101 \mathrm{MHz}, \mathrm{CDCl}_{3}\right) \delta 14.2\left(\mathrm{CH}_{3}\right), 21.6$ $\left(\mathrm{CH}_{3}\right), 61.3\left(\mathrm{CH}_{2}\right), 123.0(\mathrm{CH}), 123.1(\mathrm{CH}), 124.3(\mathrm{CH}), 125.5$ (CH), $127.2(2 \times \mathrm{CH}), 127.9(\mathrm{C}), 130.1(2 \times \mathrm{CH}), 135.6(\mathrm{C}), 136.5$ (CH), 140.6 (C), 144.7 (C), 144.9 (C), 165.9 (C); MS (ESI) $m / z 413$ $\left(\mathrm{MNa}^{+}, 100\right)$; HRMS (ESI) calcd for $\mathrm{C}_{18} \mathrm{H}_{18} \mathrm{~N}_{2} \mathrm{NaO}_{6} \mathrm{~S}\left(\mathrm{MNa}^{+}\right)$, 413.0778; found, 413.0760 .

Ethyl (2E)-3-(2'-[N-Allyl- $N$-( $p$-toluenesulfonyl)amino]-5'nitrophenyl)prop-2-enoate (9). The reaction was carried out as described for the synthesis of methyl (2E)-3- $\left(2^{\prime}-[N-\right.$ allyl- $N-(p-$ toluenesulfonyl)amino]phenyl)prop-2-enoate (5a) using ethyl $(2 E)$ 3 -(2'-[N-( $p$-toluenesulfonyl)amino $]-5^{\prime}$-nitrophenyl)prop-2-enoate $(0.020 \mathrm{~g}, 0.047 \mathrm{mmol})$. Purification by column chromatography (ethyl acetate/petroleum ether, 1:10) gave ethyl (2E)-3- $\left(2^{\prime}-[N\right.$-allyl- $N-(p-$ toluenesulfonyl)amino]-5'-nitrophenyl)prop-2-enoate (9) (0.012 g, $55 \%$ ) as a white solid. $\mathrm{Mp} 128-130{ }^{\circ} \mathrm{C} ; R_{f}=0.50$ (petroleum ether/ ethyl acetate $=2: 1)$; IR (neat) 2956, 1716, 1529, 1349, 1215, 908, 730 $\mathrm{cm}^{-1} ;{ }^{1} \mathrm{H}$ NMR $\left(400 \mathrm{MHz}, \mathrm{CDCl}_{3}\right) \delta 1.35(\mathrm{t}, J=7.1 \mathrm{~Hz}, 3 \mathrm{H}), 2.44(\mathrm{~s}$, $3 \mathrm{H}), 4.16(\mathrm{br} \mathrm{s}, 2 \mathrm{H}), 4.28(\mathrm{q}, J=7.1 \mathrm{~Hz}, 2 \mathrm{H}), 4.98(\mathrm{dd}, J=17.0,1.1$ $\mathrm{Hz}, 1 \mathrm{H}), 5.04(\mathrm{dd}, J=10.0,1.1 \mathrm{~Hz}, 1 \mathrm{H}), 5.72(\mathrm{ddt}, J=17.0,10.0,6.8$ $\mathrm{Hz}, 1 \mathrm{H}), 6.47$ (d, $J=16.1 \mathrm{~Hz}, 1 \mathrm{H}), 7.05(\mathrm{~d}, J=8.8 \mathrm{~Hz}, 1 \mathrm{H}), 7.31$ (d, $J$ $=8.2 \mathrm{~Hz}, 2 \mathrm{H}), 7.57(\mathrm{~d}, J=8.2 \mathrm{~Hz}, 2 \mathrm{H}), 7.80(\mathrm{~d}, J=16.1 \mathrm{~Hz}, 1 \mathrm{H}), 8.11$ $(\mathrm{dd}, J=8.8,2.6 \mathrm{~Hz}, 1 \mathrm{H}), 8.49(\mathrm{~d}, J=2.6 \mathrm{~Hz}, 1 \mathrm{H}) ;{ }^{13} \mathrm{C}$ NMR $(101$ $\left.\mathrm{MHz}, \mathrm{CDCl}_{3}\right) \delta 14.3\left(\mathrm{CH}_{3}\right), 21.6\left(\mathrm{CH}_{3}\right), 54.8\left(\mathrm{CH}_{2}\right), 60.9\left(\mathrm{CH}_{2}\right)$, $120.7\left(\mathrm{CH}_{2}\right), 122.3(\mathrm{CH}), 122.9(\mathrm{CH}), 124.3(\mathrm{CH}), 127.9(2 \times \mathrm{CH})$, $129.9(2 \times \mathrm{CH}), 131.1(\mathrm{CH}), 131.4(\mathrm{CH}), 134.9(\mathrm{C}), 137.6(\mathrm{C})$, $138.0(\mathrm{CH}), 143.6$ (C), 144.6 (C), 147.4 (C), 165.7 (C); MS (ESI) $m / z 453\left(\mathrm{MNa}^{+}, 100\right)$; HRMS (ESI) calcd for $\mathrm{C}_{21} \mathrm{H}_{22} \mathrm{~N}_{2} \mathrm{NaO}_{6} \mathrm{~S}$ $\left(\mathrm{MNa}^{+}\right), 453.1091$; found, 453.1073 .

(2E)-3-(2'-[N-Allyl- $N$-( $p$-toluenesulfonyl)amino]-5'-nitrophenyl)prop-2-en-1-ol $(\mathbf{6 g})$. The reaction was carried out as described for the synthesis of (2E)-3-(2'-[N-allyl- $N$ - $(p$-toluenesulfonyl)amino]phenyl)prop-2-en-1-ol (6a) using ethyl (2E)-3-(2'[ $N$-allyl- $N$-( $p$-toluenesulfonyl)amino]-5' -nitrophenyl)prop-2-enoate (9) $(0.143 \mathrm{~g}, 0.330 \mathrm{mmol})$. Purification by column chromatography (ethyl acetate/petroleum ether $=1: 2)$ gave $(2 E)-3-\left(2^{\prime}-[N\right.$-allyl- $N-(p-$ toluenesulfonyl)amino]-5' -nitrophenyl)prop-2-en-1-ol (6g) (0.110 g, $85 \%$ ) as a colorless oil. $R_{f}=0.18$ (petroleum ether/ethyl acetate = 2:1); IR (neat) 3537, 2924, 1525, 1347, 1162, $748 \mathrm{~cm}^{-1}$; ${ }^{1} \mathrm{H}$ NMR $\left(400 \mathrm{MHz}, \mathrm{CDCl}_{3}\right) \delta 2.05(\mathrm{br} \mathrm{s}, 1 \mathrm{H}), 2.45(\mathrm{~s}, 3 \mathrm{H}), 4.12(\mathrm{br} \mathrm{s}, 2 \mathrm{H})$, $4.33(\mathrm{br} \mathrm{d}, J=4.9 \mathrm{~Hz}, 2 \mathrm{H}), 4.92-5.04(\mathrm{~m}, 2 \mathrm{H}), 5.69(\mathrm{ddt}, J=17.0$, $10.1,6.8 \mathrm{~Hz}, 1 \mathrm{H}), 6.49(\mathrm{dt}, J=16.0,4.9 \mathrm{~Hz}, 1 \mathrm{H}), 6.85(\mathrm{dt}, J=16.0$, $1.6 \mathrm{~Hz}, 1 \mathrm{H}), 6.86(\mathrm{~d}, J=8.8 \mathrm{~Hz}, 1 \mathrm{H}), 7.32(\mathrm{~d}, J=8.2 \mathrm{~Hz}, 2 \mathrm{H}), 7.57$ $(\mathrm{d}, J=8.2 \mathrm{~Hz}, 2 \mathrm{H}), 7.95(\mathrm{dd}, J=8.8,2.7 \mathrm{~Hz}, 1 \mathrm{H}), 8.43(\mathrm{~d}, J=2.7 \mathrm{~Hz}$, $1 \mathrm{H}) ;{ }^{13} \mathrm{C}$ NMR $\left(101 \mathrm{MHz}, \mathrm{CDCl}_{3}\right) \delta 21.6\left(\mathrm{CH}_{3}\right), 54.7\left(\mathrm{CH}_{2}\right), 63.2$ $\left(\mathrm{CH}_{2}\right), 120.4\left(\mathrm{CH}_{2}\right), 121.7(\mathrm{CH}), 122.1(\mathrm{CH}), 124.4(\mathrm{CH}), 127.9(2$ $\times \mathrm{CH}), 129.8(2 \times \mathrm{CH}), 130.5(\mathrm{CH}), 131.5(\mathrm{CH}), 134.1(\mathrm{CH}), 135.4$ (C), 139.9 (C), 142.0 (C), 144.3 (C), 147.5 (C); MS (ESI) $\mathrm{m} / z 411$ $\left(\mathrm{MNa}^{+}, 100\right)$; HRMS (ESI) calcd for $\mathrm{C}_{19} \mathrm{H}_{20} \mathrm{~N}_{2} \mathrm{NaO}_{5} \mathrm{~S}\left(\mathrm{MNa}^{+}\right)$, 411.0985; found, 411.0970 .

$\mathrm{N}$-( $p$-Toluenesulfonyl)-5-(2',2',2'-trichloromethylcarbonylamino)-2,5-dihydro-1H-benzo[b]azepine (10a). (2E)-3-(2'-[NAllyl- $N$-( $p$-toluenesulfonyl)amino]phenyl)prop-2-en-1-ol (6a) (0.313 g, $0.911 \mathrm{mmol})$ was dissolved in dichloromethane $(45 \mathrm{~mL})$ and cooled to $0{ }^{\circ} \mathrm{C}$ under argon with stirring. Trichloroacetonitrile $(0.137 \mathrm{~mL}$, $1.37 \mathrm{mmol}$ ) was added to the solution, followed by 1,8 -diazabicyclo[5.4.0]undec-7-ene $(0.0685 \mathrm{~mL}, 0.460 \mathrm{mmol})$, and the reaction was allowed to warm to room temperature over $2 \mathrm{~h}$. The reaction mixture was filtered through a short pad of alumina (neutral, Brockman V) with diethyl ether $(150 \mathrm{~mL})$ and concentrated in vacuo to yield the crude allylic trichloroacetimidate as a yellow oil. This was used without further purification. The allylic trichloroacetimidate was transferred to a dry Schlenk tube containing a stirrer bar and potassium carbonate $(0.0300 \mathrm{~g}, 5 \mathrm{mg} / \mathrm{mL})$ to which $p$-xylene $(6 \mathrm{~mL})$ was then added. The tube was purged with argon, sealed, and heated to $160{ }^{\circ} \mathrm{C}$ for $24 \mathrm{~h}$. The reaction mixture was allowed to cool to room temperature, and Grubbs' second generation catalyst $(0.0391 \mathrm{~g}, 0.0460$ mmol) and $p$-xylene $(51 \mathrm{~mL})$ were added. The reaction mixture was heated to $60{ }^{\circ} \mathrm{C}$ for $18 \mathrm{~h}$. The reaction mixture was concentrated in 
vacuo and purified by column chromatography (diethyl ether/ petroleum ether $=1: 3)$ to give $N$ - $(p$-toluenesulfonyl $)-5-\left(2^{\prime}, 2^{\prime}, 2^{\prime}\right.$ trichloromethylcarbonylamino)-2,5-dihydro- $1 H$-benzo $[b]$ azepine (10a) $(0.339 \mathrm{~g}, 81 \%)$ as a white solid. Mp $160-163{ }^{\circ} \mathrm{C}$ (decomposition); $R_{f}=0.28$ (diethyl ether/petroleum ether $=1: 1$ ); IR (neat) 3337, 2925, 1701, 1496, 1341, 1159, 906, $727 \mathrm{~cm}^{-1} ;{ }^{1} \mathrm{H}$ NMR (400 MHz, $\left.\mathrm{CDCl}_{3}\right) \delta 2.47(\mathrm{~s}, 3 \mathrm{H}), 3.86(\mathrm{br} \mathrm{s}, 1 \mathrm{H}), 4.66$ (br s, $1 \mathrm{H}), 5.58(\mathrm{brt}, J=7.7 \mathrm{~Hz}, 1 \mathrm{H}), 5.84(\mathrm{br} \mathrm{d}, J=9.0 \mathrm{~Hz}, 1 \mathrm{H}), 6.04(\mathrm{br} \mathrm{s}$, $1 \mathrm{H}), 6.82(\mathrm{br} \mathrm{s}, 1 \mathrm{H}), 7.23(\mathrm{td}, J=8.4,1.6 \mathrm{~Hz}, 1 \mathrm{H}), 7.31(\mathrm{td}, J=8.4$, $1.3 \mathrm{~Hz}, 1 \mathrm{H}), 7.36(\mathrm{~d}, J=8.2 \mathrm{~Hz}, 2 \mathrm{H}), 7.42(\mathrm{br} \mathrm{d}, J=8.4 \mathrm{~Hz}, 1 \mathrm{H}), 7.77$ $(\mathrm{d}, J=8.2 \mathrm{~Hz}, 2 \mathrm{H}), 8.37(\mathrm{br} \mathrm{s}, 1 \mathrm{H}) ;{ }^{13} \mathrm{C} \mathrm{NMR}\left(101 \mathrm{MHz}, \mathrm{CDCl}_{3}\right) \delta$ $21.6\left(\mathrm{CH}_{3}\right), 49.0\left(\mathrm{CH}_{2}\right), 52.7(\mathrm{CH}), 92.5(\mathrm{C}), 125.8(\mathrm{CH}), 127.4(2$ $\times \mathrm{CH}), 128.2(\mathrm{CH}), 129.3(\mathrm{CH}), 129.7(\mathrm{CH}), 130.0(2 \times \mathrm{CH}), 130.8$ ( $2 \times \mathrm{CH}), 137.7$ (C), 138.1 (C), 139.2 (C), 144.2 (C), 161.4 (C); MS (ESI) $m / z \quad 481 \quad\left(\mathrm{MNa}^{+}, 49\right)$; HRMS (ESI) calcd for $\mathrm{C}_{19} \mathrm{H}_{17}{ }^{35} \mathrm{Cl}_{3} \mathrm{~N}_{2} \mathrm{NaO}_{3} \mathrm{~S}\left(\mathrm{MNa}^{+}\right)$, 480.9918; found, 480.9904.

7-Methyl- $N$-( $p$-toluenesulfonyl)-5-(2', $2^{\prime}, 2^{\prime}$-trichloromethylcarbonylamino)-2,5-dihydro-1 $\mathrm{H}$-benzo[b]azepine (10b). The reaction was carried out as described for the synthesis of $N$ - $(p$ toluenesulfonyl)-5-( $2^{\prime}, 2^{\prime}, 2^{\prime}$-trichloromethylcarbonylamino $)-2,5$-dihydro- $1 H$-benzo[b]azepine (10a) using (2E)-3- $\left(2^{\prime}-[N\right.$-allyl- $N-(p-$ toluenesulfonyl)amino]-5'-methylphenyl)prop-2-en-1-ol (6b) (0.170 g, $0.480 \mathrm{mmol}$ ). Purification by column chromatography (diethyl ether/petroleum ether $=1: 3)$ gave 7-methyl- $N$ - $(p$-toluenesulfonyl $)-5$ $\left(2^{\prime}, 2^{\prime}, 2^{\prime}\right.$-trichloromethylcarbonylamino)-2,5-dihydro- $1 H$-benzo[b]azepine $(10 \mathrm{~b})(0.179 \mathrm{~g}, 80 \%)$ as a white solid. Mp $174-176^{\circ} \mathrm{C} ; R_{f}=$ 0.30 (diethyl ether/petroleum ether $=1: 1$ ); IR (neat) 3333, 2923, 1701, 1505, 1340, 1155, 1112, 909, $727 \mathrm{~cm}^{-1}$; ${ }^{1} \mathrm{H}$ NMR (400 MHz, $\left.\mathrm{CDCl}_{3}\right) \delta 2.33(\mathrm{~s}, 3 \mathrm{H}), 2.47(\mathrm{~s}, 3 \mathrm{H}), 3.82(\mathrm{br} \mathrm{s}, 1 \mathrm{H}), 4.67(\mathrm{br} \mathrm{s}, 1 \mathrm{H})$, $5.53(\mathrm{brt}, J=7.8 \mathrm{~Hz}, 1 \mathrm{H}), 5.84(\mathrm{br} \mathrm{d}, J=8.6 \mathrm{~Hz}, 1 \mathrm{H}), 6.04(\mathrm{br} \mathrm{s}, 1 \mathrm{H})$, 6.67 (br s, $1 \mathrm{H}), 7.02(\mathrm{dd}, J=8.1,1.4 \mathrm{~Hz}, 1 \mathrm{H}), 7.23$ (br s, $1 \mathrm{H}), 7.36$ (d, $J=8.2 \mathrm{~Hz}, 2 \mathrm{H}), 7.76(\mathrm{~d}, J=8.2 \mathrm{~Hz}, 2 \mathrm{H}), 8.43(\mathrm{br} \mathrm{s}, 1 \mathrm{H}) ;{ }^{13} \mathrm{C} \mathrm{NMR}$ $\left(101 \mathrm{MHz}, \mathrm{CDCl}_{3}\right) \delta 21.1\left(\mathrm{CH}_{3}\right), 21.6\left(\mathrm{CH}_{3}\right), 49.1\left(\mathrm{CH}_{2}\right), 52.7$ (CH), $92.5(\mathrm{C}), 125.8(\mathrm{CH}), 127.4(2 \times \mathrm{CH}), 127.9(\mathrm{CH}), 130.0(2$ $\times \mathrm{CH}), 130.2(2 \times \mathrm{CH}), 130.9(\mathrm{CH}), 135.4(\mathrm{C}), 137.8(\mathrm{C}), 138.8$ (C), 139.4 (C), 144.1 (C), 161.4 (C); MS (ESI) $\mathrm{m} / z 495\left(\mathrm{MNa}^{+}, 48\right)$; HRMS (ESI) calcd for $\mathrm{C}_{20} \mathrm{H}_{19}{ }^{35} \mathrm{Cl}_{3} \mathrm{~N}_{2} \mathrm{NaO}_{3} \mathrm{~S}\left(\mathrm{MNa}^{+}\right)$, 495.0074; found, 495.0053.

7-Methoxy- $N$-(p-toluenesulfonyl)-5-(2', $2^{\prime}, 2^{\prime}$-trichloromethylcarbonylamino)-2,5-dihydro- $1 \mathrm{H}$-benzo[b]azepine (10c). The reaction was carried out as described for the synthesis of $\mathrm{N}-(p-$ toluenesulfonyl)-5-( $\left(2^{\prime}, 2^{\prime}, 2^{\prime}\right.$-trichloromethylcarbonylamino $)-2,5$-dihydro- $1 H$-benzo[b]azepine (10a) using (2E)-3-(2'-[N-allyl- $N-(p-$ toluenesulfonyl)amino]-5'-methoxyphenyl)prop-2-en-1-ol (6c) $(0.076 \mathrm{~g}, 0.20 \mathrm{mmol})$. Purification by column chromatography (diethyl ether/petroleum ether $=1: 3)$ gave 7 -methoxy- $N$ - $(p$ toluenesulfonyl)-5-(2',2',2'-trichloromethylcarbonylamino $)$-2,5-dihydro- $1 H$-benzo $[b]$ azepine $(10 \mathrm{c})(0.079 \mathrm{~g}, 79 \%)$ as a white solid. Mp 190-195 ${ }^{\circ} \mathrm{C}$ (decomposition); $R_{f}=0.20$ (diethyl ether/petroleum ether = 1:1); IR (neat) 3337, 2935, 1701, 1502, 1215, 1156, $749 \mathrm{~cm}^{-1}$; ${ }^{1} \mathrm{H}$ NMR $\left(400 \mathrm{MHz}, \mathrm{CDCl}_{3}\right) \delta 2.46(\mathrm{~s}, 3 \mathrm{H}), 3.61-3.84(\mathrm{~m}, 4 \mathrm{H}), 4.72$ (br s, $1 \mathrm{H}), 5.51($ br t, $J=7.6 \mathrm{~Hz}, 1 \mathrm{H}), 5.85($ br s, $1 \mathrm{H}), 6.05($ br s, $1 \mathrm{H})$, $6.64(\mathrm{br} \mathrm{s}, 1 \mathrm{H}), 6.71(\mathrm{dd}, J=8.6,2.8 \mathrm{~Hz}, 1 \mathrm{H}), 6.93(\mathrm{br} \mathrm{s}, 1 \mathrm{H}), 7.35(\mathrm{~d}$, $J=8.2 \mathrm{~Hz}, 2 \mathrm{H}), 7.74(\mathrm{~d}, J=8.2 \mathrm{~Hz}, 2 \mathrm{H}), 8.58(\mathrm{br} \mathrm{s}, 1 \mathrm{H}) ;{ }^{13} \mathrm{C}$ NMR $\left(101 \mathrm{MHz}, \mathrm{CDCl}_{3}\right) \delta 21.6\left(\mathrm{CH}_{3}\right), 49.2\left(\mathrm{CH}_{2}\right), 52.9(\mathrm{CH}), 55.6$ $\left(\mathrm{CH}_{3}\right), 92.5(\mathrm{C}), 114.9(\mathrm{CH}), 125.5(\mathrm{CH}), 127.4(2 \times \mathrm{CH}), 129.2$ $(\mathrm{CH}), 130.0(2 \times \mathrm{CH}), 130.4(\mathrm{CH}), 131.2(\mathrm{CH}), 137.7(\mathrm{C}), 140.5$ (C), 144.1 (C), $159.7(2 \times \mathrm{C}), 161.4(\mathrm{C})$; MS (ESI) $m / z 513\left(\mathrm{MNa}^{+}\right.$, 51); HRMS (ESI) calcd for $\mathrm{C}_{20} \mathrm{H}_{19}{ }^{35} \mathrm{Cl}_{2}^{37} \mathrm{ClN}_{2} \mathrm{NaO}_{4} \mathrm{~S}\left(\mathrm{MNa}^{+}\right)$, 512.9994; found, 512.9973.

7-Fluoro- $N$-( $p$-toluenesulfonyl)-5-(2',2',2'-trichloromethylcarbonylamino)-2,5-dihydro- $1 \mathrm{H}$-benzo[b]azepine (10d). The reaction was carried out as described for the synthesis of $N$ - $(p$ toluenesulfonyl)-5-( $\left(2^{\prime}, 2^{\prime}, 2^{\prime}\right.$-trichloromethylcarbonylamino $)-2,5$-dihydro- $1 H$-benzo $[b]$ azepine (10a) using (2E)-3- $\left(2^{\prime}-[N\right.$-allyl- $N-(p-$ toluenesulfonyl)amino]-5'-fluorophenyl)prop-2-en-1-ol (6d) (0.189 g, $0.520 \mathrm{mmol}$ ). Purification by column chromatography (diethyl ether/petroleum ether $=1: 3)$ gave 7-fluoro- $N$ - $(p$-toluenesulfonyl $)-5$ $\left(2^{\prime}, 2^{\prime}, 2^{\prime}\right.$-trichloromethylcarbonylamino)-2,5-dihydro- $1 H$-benzo[b]azepine (10d) $(0.204 \mathrm{~g}, 82 \%)$ as a white solid. Mp 181-183 ${ }^{\circ} \mathrm{C} ; R_{f}=$
0.25 (petroleum ether/diethyl ether $=3: 1$ ); IR (neat) 3333, 3034, $1705,1503,1344,1159,907,729 \mathrm{~cm}^{-1}$; ${ }^{1} \mathrm{H}$ NMR $\left(400 \mathrm{MHz}, \mathrm{CDCl}_{3}\right)$ $\delta 2.47(\mathrm{~s}, 3 \mathrm{H}), 3.81(\mathrm{br} \mathrm{s}, 1 \mathrm{H}), 4.62(\mathrm{br} \mathrm{s}, 1 \mathrm{H}), 5.52(\mathrm{br} \mathrm{t}, J=7.4 \mathrm{~Hz}$, $1 \mathrm{H}), 5.85(\mathrm{br} \mathrm{s}, 1 \mathrm{H}), 5.98(\mathrm{br} \mathrm{s}, 1 \mathrm{H}), 6.81(\mathrm{br} \mathrm{s}, 1 \mathrm{H}), 6.91(\mathrm{td}, J=8.2$, $2.9 \mathrm{~Hz}, 1 \mathrm{H}), 7.12(\mathrm{br} \mathrm{s}, 1 \mathrm{H}), 7.36(\mathrm{~d}, J=8.2 \mathrm{~Hz}, 2 \mathrm{H}), 7.75(\mathrm{~d}, J=8.2$ $\mathrm{Hz}, 2 \mathrm{H}), 8.40(\mathrm{br} \mathrm{s}, 1 \mathrm{H}) ;{ }^{13} \mathrm{C} \mathrm{NMR}\left(101 \mathrm{MHz}, \mathrm{CDCl}_{3}\right) \delta 21.6\left(\mathrm{CH}_{3}\right)$, $48.9\left(\mathrm{CH}_{2}\right), 52.2(\mathrm{CH}), 92.4(\mathrm{C}), 116.1(\mathrm{CH}), 116.4(\mathrm{CH}), 125.4$ $(\mathrm{CH}), 127.4(2 \times \mathrm{CH}), 130.1(3 \times \mathrm{CH}), 131.1(\mathrm{CH}), 133.9(\mathrm{C})$, 137.3 (C), 141.6 (C), 144.4 (C), 161.4 (C), $162.1\left(\mathrm{~d},{ }^{1} J_{\mathrm{CF}}=250.6 \mathrm{~Hz}\right.$, C); MS (ESI) $m / z 499\left(\mathrm{MNa}^{+}, 49\right)$; HRMS (ESI) calcd for $\mathrm{C}_{19} \mathrm{H}_{16}{ }^{35} \mathrm{Cl}_{3} \mathrm{FN}_{2} \mathrm{NaO}_{3} \mathrm{~S}\left(\mathrm{MNa}^{+}\right)$, 498.9823; found, 498.9809 .

8-Fluoro- $N$-( $p$-toluenesulfonyl)-5- $\left(2^{\prime}, 2^{\prime}, 2^{\prime}\right.$-trichloromethylcarbonylamino)-2,5-dihydro-1 $H$-benzo[b]azepine (10e). The reaction was carried out as described for the synthesis of $N-(p$ toluenesulfonyl)-5-( $2^{\prime}, 2^{\prime}, 2^{\prime}$-trichloromethylcarbonylamino)-2,5-dihydro- $1 H$-benzo $[b]$ azepine (10a) using (2E)-3- $\left(2^{\prime}-[N\right.$-allyl- $N-(p-$ toluenesulfonyl)amino]-4'-fluorophenyl)prop-2-en-1-ol (6e) (0.222 g, $0.610 \mathrm{mmol}$ ). Purification by column chromatography (diethyl ether/petroleum ether $=1: 3)$ gave 8 -fluoro- $N$ - $(p$-toluenesulfonyl $)-5$ $\left(2^{\prime}, 2^{\prime}, 2^{\prime}\right.$-trichloromethylcarbonylamino $)-2,5$-dihydro- $1 H$-benzo[ $\left.b\right]$ azepine $(10 \mathrm{e})(0.269 \mathrm{~g}, 92 \%)$ as a white solid. Mp $147-149{ }^{\circ} \mathrm{C} ; R_{f}=$ 0.28 (diethyl ether/petroleum ether =1:1); IR (neat) 3340, 2925, 1704, 1599, 1501, 1343, 1160, 909, $731 \mathrm{~cm}^{-1} ;{ }^{1} \mathrm{H}$ NMR (400 MHz, $\left.\mathrm{CDCl}_{3}\right) \delta 2.48(\mathrm{~s}, 3 \mathrm{H}), 3.86(\mathrm{br} \mathrm{d}, J=17.8 \mathrm{~Hz}, 1 \mathrm{H}), 4.62(\mathrm{br} \mathrm{d}, J=$ $17.8 \mathrm{~Hz}, 1 \mathrm{H}$ ), 5.56 (br t, $J=7.8 \mathrm{~Hz}, 1 \mathrm{H}$ ), 5.85 (ddd, $J=11.4,4.5,1.8$ $\mathrm{Hz}, 1 \mathrm{H}), 6.02(\mathrm{dd}, J=11.4,7.8 \mathrm{~Hz}, 1 \mathrm{H}), 6.55\left(\mathrm{br} \mathrm{d},{ }^{3} J_{\mathrm{HF}}=8.0 \mathrm{~Hz}\right.$, $1 \mathrm{H}), 7.02(\mathrm{td}, J=8.2,2.6 \mathrm{~Hz}, 1 \mathrm{H}), 7.38(\mathrm{~d}, J=8.2 \mathrm{~Hz}, 2 \mathrm{H}), 7.41(\mathrm{dd}$, $\left.J=8.2,{ }^{4} J_{\mathrm{HF}}=6.4 \mathrm{~Hz}, 1 \mathrm{H}\right), 7.77(\mathrm{~d}, J=8.2 \mathrm{~Hz}, 2 \mathrm{H}), 8.24(\mathrm{br} \mathrm{s}, 1 \mathrm{H})$; ${ }^{13} \mathrm{C}$ NMR $\left(101 \mathrm{MHz}, \mathrm{CDCl}_{3}\right) \delta 21.7\left(\mathrm{CH}_{3}\right), 48.8\left(\mathrm{CH}_{2}\right), 52.1(\mathrm{CH})$, $92.4(\mathrm{C}), 115.6\left(\mathrm{~d},{ }^{2} J_{C F}=22.9 \mathrm{~Hz}, \mathrm{CH}\right), 116.2\left(\mathrm{~d},{ }^{2} J_{C F}=21.0 \mathrm{~Hz}\right.$, $\mathrm{CH}), 125.6(\mathrm{CH}), 127.4(2 \times \mathrm{CH}), 130.2(2 \times \mathrm{CH}), 130.7(\mathrm{CH})$, $132.0(\mathrm{CH}), 135.3\left(\mathrm{~d},{ }^{4} J_{\mathrm{CF}}=3.5 \mathrm{~Hz}, \mathrm{C}\right), 137.2(\mathrm{C}), 139.4\left(\mathrm{~d},{ }^{3} J_{\mathrm{CF}}=\right.$ $9.9 \mathrm{~Hz}, \mathrm{C}), 144.6$ (C), 161.4 (C), $162.5\left(\mathrm{~d},{ }^{1} J_{\mathrm{CF}}=230.0 \mathrm{~Hz}, \mathrm{C}\right) ; \mathrm{MS}$ (ESI) $m / z 499\left(\mathrm{MNa}^{+}, 49\right)$; HRMS (ESI) calcd for $\mathrm{C}_{19} \mathrm{H}_{16}{ }^{35} \mathrm{Cl}_{3} \mathrm{FN}_{2} \mathrm{NaO}_{3} \mathrm{~S}\left(\mathrm{MNa}^{+}\right)$, 498.9823; found, 498.9804.

7-Chloro- $N$-( $p$-toluenesulfonyl)-5-(2', $2^{\prime}, 2^{\prime}$-trichloromethylcarbonylamino)-2,5-dihydro-1 $\mathrm{H}$-benzo[b]azepine (10f). The reaction was carried out as described for the synthesis of $N$ - $(p$ toluenesulfonyl)-5-( $2^{\prime}, 2^{\prime}, 2^{\prime}$-trichloromethylcarbonylamino $)-2,5$-dihydro- $1 H$-benzo[b]azepine (10a) using (2E)-3- $\left(2^{\prime}-[N\right.$-allyl- $N-(p-$ toluenesulfonyl)amino]-5' -chlorophenyl)prop-2-en-1-ol (6f) (0.290 g, $0.770 \mathrm{mmol}$ ). The RCM step was heated to $60{ }^{\circ} \mathrm{C}$ for $24 \mathrm{~h}$. Purification by column chromatography (diethyl ether/petroleum ether = 1:3) gave 7-chloro- $N$ - $(p$-toluenesulfonyl $)-5-\left(2^{\prime}, 2^{\prime}, 2^{\prime}\right.$-trichloromethylcarbonylamino)-2,5-dihydro-1H-benzo[b]azepine (10f) $(0.300$ $\mathrm{g}, 79 \%$ ) as a white solid. Mp $158-160{ }^{\circ} \mathrm{C} ; R_{f}=0.25$ (diethyl ether/ petroleum ether =1:1); IR (neat) 3341, 2925, 1705, 1495, 1343, 1159, 908, $730 \mathrm{~cm}^{-1} ;{ }^{1} \mathrm{H}$ NMR $\left(400 \mathrm{MHz}, \mathrm{CDCl}_{3}\right) \delta 2.47(\mathrm{~s}, 3 \mathrm{H}), 3.88(\mathrm{br}$ s, $1 \mathrm{H}), 4.60($ br s, $1 \mathrm{H}), 5.51$ (br t, $J=7.6 \mathrm{~Hz}, 1 \mathrm{H}), 5.84(\mathrm{br} \mathrm{d}, J=9.0$ $\mathrm{Hz}, 1 \mathrm{H}), 5.97$ (br s, $1 \mathrm{H}), 6.79$ (br s, $1 \mathrm{H}), 7.20$ (dd, $J=8.4,2.4 \mathrm{~Hz}$, $1 \mathrm{H}), 7.36(\mathrm{~d}, J=8.2 \mathrm{~Hz}, 2 \mathrm{H}), 7.40(\mathrm{br} \mathrm{s}, 1 \mathrm{H}), 7.75(\mathrm{~d}, J=8.2 \mathrm{~Hz}$, $2 \mathrm{H}), 8.26($ br s, $1 \mathrm{H}) ;{ }^{13} \mathrm{C}$ NMR $\left(101 \mathrm{MHz}, \mathrm{CDCl}_{3}\right) \delta 21.7\left(\mathrm{CH}_{3}\right)$, $48.9\left(\mathrm{CH}_{2}\right), 52.1(\mathrm{CH}), 92.3(\mathrm{C}), 125.4(\mathrm{CH}), 127.4(2 \times \mathrm{CH}), 129.6$ $(2 \times \mathrm{CH}), 130.2(2 \times \mathrm{CH}), 131.0(2 \times \mathrm{CH}), 134.9(\mathrm{C}), 136.5(\mathrm{C})$, 137.2 (C), 141.0 (C), 144.5 (C), 161.4 (C); MS (ESI) $\mathrm{m} / z 515$ $\left(\mathrm{MNa}^{+}, 42\right)$; HRMS (ESI) calcd for $\mathrm{C}_{19} \mathrm{H}_{16}{ }^{35} \mathrm{Cl}_{4} \mathrm{~N}_{2} \mathrm{NaO}_{3} \mathrm{~S}\left(\mathrm{MNa}^{+}\right)$, 514.9528; found, 514.9515 .

7-Nitro- $N$-( $p$-toluenesulfonyl)-5-(2', $2^{\prime}, 2^{\prime}$-trichloromethylcarbonylamino)-2,5-dihydro- $1 H$-benzo[b]azepine $(10 \mathrm{~g})$. The reaction was carried out as described for the synthesis of $N$ - $(p$ toluenesulfonyl)-5-( $2^{\prime}, 2^{\prime}, 2^{\prime}$-trichloromethylcarbonylamino $)$-2,5-dihydro- $1 H$-benzo $[b]$ azepine (10a) using (2E)-3- $\left(2^{\prime}-[N\right.$-allyl- $N-(p-$ toluenesulfonyl)amino]-5'-nitrophenyl)prop-2-en-1-ol (6g) (0.084 g, $0.22 \mathrm{mmol})$. The Overman rearrangement was heated to $160{ }^{\circ} \mathrm{C}$ for $43 \mathrm{~h}$, and the RCM step was heated to $60^{\circ} \mathrm{C}$ for $31 \mathrm{~h}$. Purification by column chromatography (diethyl ether/petroleum ether $=1: 3$ ) gave 7 nitro- $N$ - ( $p$-toluenesulfonyl)-5- $\left(2^{\prime}, 2^{\prime}, 2^{\prime}\right.$-trichloromethylcarbonylamino)-2,5-dihydro- $1 H$-benzo $[b]$ azepine $(10 \mathrm{~g})(0.053 \mathrm{~g}, 49 \%)$ as a white solid. Mp $180-185{ }^{\circ} \mathrm{C}$ (decomposition); $R_{f}=0.28$ (diethyl ether/petroleum ether =1:1); IR (neat) 3335, 3020, 1709, 1592, 1530, 
1350, 1215, 1161, $749 \mathrm{~cm}^{-1} ;{ }^{1} \mathrm{H}$ NMR (400 MHz, $\left.\mathrm{CDCl}_{3}\right) \delta 2.49(\mathrm{~s}$, $3 \mathrm{H}), 4.03(\mathrm{br} \mathrm{d}, J=18.5 \mathrm{~Hz}, 1 \mathrm{H}), 4.54(\mathrm{br} \mathrm{d}, J=18.5 \mathrm{~Hz}, 1 \mathrm{H}), 5.64$ (br t, $J=7.2 \mathrm{~Hz}, 1 \mathrm{H}), 5.86($ br d, $J=11.4 \mathrm{~Hz}, 1 \mathrm{H}), 5.95-6.02(\mathrm{~m}$, $1 \mathrm{H}), 7.18(\mathrm{~d}, J 8.6 \mathrm{~Hz}, 1 \mathrm{H}), 7.41(\mathrm{~d}, J=8.2 \mathrm{~Hz}, 2 \mathrm{H}), 7.79(\mathrm{~d}, J=8.2$ $\mathrm{Hz}, 2 \mathrm{H}), 7.98(\mathrm{br} \mathrm{s}, 1 \mathrm{H}), 8.13(\mathrm{dd}, J=8.6,2.6 \mathrm{~Hz}, 1 \mathrm{H}), 8.29(\mathrm{~d}, J=$ $2.6 \mathrm{~Hz}, 1 \mathrm{H}) ;{ }^{13} \mathrm{C} \mathrm{NMR}\left(101 \mathrm{MHz}, \mathrm{CDCl}_{3}\right) \delta 21.7\left(\mathrm{CH}_{3}\right), 48.7\left(\mathrm{CH}_{2}\right)$, 51.8 (CH), $92.1(\mathrm{C}), 124.5(2 \times \mathrm{CH}), 125.1(\mathrm{CH}), 127.4(2 \times \mathrm{CH})$, $129.6(\mathrm{CH}), 130.4(2 \times \mathrm{CH}), 130.7(\mathrm{CH}), 136.8(\mathrm{C}), 141.1(\mathrm{C})$, 143.8 (C), 145.0 (C), 147.4 (C), 161.5 (C); MS (ESI) $m / z 526$ $\left(\mathrm{MNa}^{+}, 49\right)$; HRMS (ESI) calcd for $\mathrm{C}_{19} \mathrm{H}_{16}{ }^{35} \mathrm{Cl}_{3} \mathrm{~N}_{3} \mathrm{NaO}_{5} \mathrm{~S}\left(\mathrm{MNa}^{+}\right)$, 525.9768; found, 525.9761.

5-tert-Butoxycarbonylamino- $\mathrm{N}$-(p-toluenesulfonyl)-2,5-dihydro-1H-benzo[b]azepine (11). Sodium hydroxide $(2 \mathrm{M}, 5 \mathrm{~mL})$ was added to a solution of $N$-( $p$-toluenesulfonyl $)-5-\left(2^{\prime}, 2^{\prime}, 2^{\prime}\right.$-trichloromethylcarbonylamino)-2,5-dihydro- $1 H$-benzo[b]azepine (10a) $(0.165$ g, $0.359 \mathrm{mmol})$ in methanol $(3 \mathrm{~mL})$ at $60{ }^{\circ} \mathrm{C}$ and stirred for $18 \mathrm{~h}$. The mixture was allowed to cool to room temperature, and then di-tertbutyl dicarbonate $(0.393 \mathrm{~g}, 1.80 \mathrm{mmol})$ was added. The reaction mixture was stirred for a further $24 \mathrm{~h}$. The reaction mixture was extracted with ethyl acetate $(3 \times 5 \mathrm{~mL})$, dried $\left(\mathrm{MgSO}_{4}\right)$, filtered, and concentrated in vacuo. Purification by column chromatography (ethyl acetate/petroleum ether $=1: 20)$ gave 5-tert-butoxycarbonylamino- $N$ ( $p$-toluenesulfonyl)-2,5-dihydro-1H-benzo[b]azepine (11) (0.108 g, $73 \%)$ as a white solid. $\mathrm{Mp} 149-151{ }^{\circ} \mathrm{C}$ (decomposition); $R_{f}=0.28$ (petroleum ether/ethyl acetate $=2: 1$ ); IR (neat) 3393, 2978, 1698, $1494,1343,1159,908,728 \mathrm{~cm}^{-1}$; ${ }^{1} \mathrm{H}$ NMR $\left(400 \mathrm{MHz}, \mathrm{CDCl}_{3}\right) \delta 1.44$ (s, 9H), $2.45(\mathrm{~s}, 3 \mathrm{H}), 4.14($ br s, $1 \mathrm{H}), 4.35($ br s, $1 \mathrm{H}), 5.33($ br t, $J=$ $7.2 \mathrm{~Hz}, 1 \mathrm{H}), 5.50(\mathrm{br} \mathrm{s}, 1 \mathrm{H}), 5.61(\mathrm{br} \mathrm{d}, J=10.7 \mathrm{~Hz}, 1 \mathrm{H}), 5.81(\mathrm{br} \mathrm{s}$, $1 \mathrm{H}), 7.05(\mathrm{~d}, J=7.6 \mathrm{~Hz}, 1 \mathrm{H}), 7.21(\mathrm{td}, J=7.6,1.6 \mathrm{~Hz}, 1 \mathrm{H}), 7.27-7.36$ $(\mathrm{m}, 4 \mathrm{H}), 7.77$ (br d, $J=8.2 \mathrm{~Hz}, 2 \mathrm{H}) ;{ }^{13} \mathrm{C} \mathrm{NMR}\left(126 \mathrm{MHz}, \mathrm{CDCl}_{3}\right) \delta$ $21.6\left(\mathrm{CH}_{3}\right), 28.4\left(3 \times \mathrm{CH}_{3}\right), 48.9\left(\mathrm{CH}_{2}\right), 51.4(\mathrm{CH}), 79.5(\mathrm{C}), 127.3$ $(2 \times \mathrm{CH}), 127.8(\mathrm{CH}), 128.5(3 \times \mathrm{CH}), 128.7(2 \times \mathrm{CH}), 129.9(2 \times$ CH), 137.6 (C), 138.0 (C), 141.4 (C), 143.8 (C), 154.9 (C); MS (ESI) $m / z 437\left(\mathrm{MNa}^{+}, 100\right)$; HRMS (ESI) calcd for $\mathrm{C}_{22} \mathrm{H}_{26} \mathrm{~N}_{2} \mathrm{NaO}_{4} \mathrm{~S}$ $\left(\mathrm{MNa}^{+}\right)$, 437.1505; found, 437.1486.

5-tert-Butoxycarbonylamino-2,3,4,5-tetrahydro- $1 \mathrm{H}$-benzo[b]azepine (12). ${ }^{3 \mathrm{C}}$ Palladium on charcoal $(10 \%, 0.017 \mathrm{~g})$ was added to a solution of 5-tert-butoxycarbonylamino- $N$-( $p$-toluenesulfonyl)-2,5dihydro- $1 H$-benzo[ $b$ ]azepine (11) $(0.057 \mathrm{~g}, 0.14 \mathrm{mmol})$ in ethyl acetate $(4 \mathrm{~mL})$. The mixture was stirred under an atmosphere of hydrogen at $60{ }^{\circ} \mathrm{C}$ for $17 \mathrm{~h}$. The reaction mixture was filtered through a short pad of Celite with diethyl ether $(50 \mathrm{~mL})$ and concentrated in vacuo to give 5-tert-butoxycarbonylamino- $N$-( $p$-toluenesulfonyl)2,3,4,5-tetrahydro- $1 \mathrm{H}$-benzo[ $b]$ azepine $(0.050 \mathrm{~g})$ as a white solid. 5tert-Butoxycarbonylamino- $N$-( $p$-toluenesulfonyl)-2,3,4,5-tetrahydro$1 H$-benzo[ $b$ ]azepine $(0.050 \mathrm{~g}, 0.12 \mathrm{mmol})$ was dissolved in methanol $(5 \mathrm{~mL})$, and magnesium turnings $(0.082 \mathrm{~g}, 3.4 \mathrm{mmol})$ were added. The mixture was heated under reflux for $4 \mathrm{~h}$. The reaction mixture was cooled to $0{ }^{\circ} \mathrm{C}$, and $1 \mathrm{M}$ hydrochloric acid solution $(10 \mathrm{~mL})$ was added dropwise. The solution was extracted with ethyl acetate $(3 \times 10$ $\mathrm{mL})$, dried $\left(\mathrm{MgSO}_{4}\right)$, and concentrated in vacuo. Purification by column chromatography using (ethyl acetate/petroleum ether $=1: 20$ ) gave 5-tert-butoxycarbonylamino-2,3,4,5-tetrahydro- $1 H$-benzo[b]azepine (12) $(0.032 \mathrm{~g}, 88 \%)$ as a white solid. Mp $151-153{ }^{\circ} \mathrm{C}$ (lit. ${ }^{3 \mathrm{c}} 153-154{ }^{\circ} \mathrm{C}$ ); $R_{f}=0.45$ (petroleum ether/ethyl acetate $=2: 1$ ); ${ }^{1} \mathrm{H}$ NMR (400 MHz, $\left.\mathrm{CDCl}_{3}\right) \delta 1.42(\mathrm{~s}, 9 \mathrm{H}), 1.55-1.80(\mathrm{~m}, 2 \mathrm{H})$, $1.94-2.21(\mathrm{~m}, 2 \mathrm{H}), 2.83(\mathrm{td}, J=12.8,2.0 \mathrm{~Hz}, 1 \mathrm{H}), 3.21-3.35(\mathrm{~m}$, $1 \mathrm{H}), 3.61(\mathrm{br} \mathrm{s}, 1 \mathrm{H}), 4.90(\mathrm{t}, J=8.1 \mathrm{~Hz}, 1 \mathrm{H}), 5.72(\mathrm{br} \mathrm{d}, J=8.1 \mathrm{~Hz}$, $1 \mathrm{H}), 6.73(\mathrm{dd}, J=7.3,1.1 \mathrm{~Hz}, 1 \mathrm{H}), 6.89(\mathrm{td}, J=7.3,1.1 \mathrm{~Hz}, 1 \mathrm{H}), 7.08$ $(\mathrm{td}, J=7.3,1.6 \mathrm{~Hz}, 1 \mathrm{H}), 7.23(\mathrm{br} \mathrm{d}, J=7.3 \mathrm{~Hz}, 1 \mathrm{H}) ;{ }^{13} \mathrm{C} \mathrm{NMR}(101$ $\left.\mathrm{MHz}, \mathrm{CDCl}_{3}\right) \delta 25.5\left(\mathrm{CH}_{2}\right), 28.5\left(3 \times \mathrm{CH}_{3}\right), 30.9\left(\mathrm{CH}_{2}\right), 49.1$ $\left(\mathrm{CH}_{2}\right), 55.1(\mathrm{CH}), 79.0(\mathrm{C}), 120.5(\mathrm{CH}), 121.9(\mathrm{CH}), 128.0(\mathrm{CH})$, 130.0 (CH), 133.7 (C), 149.1 (C), 155.2 (C); MS (ESI) $m / z 285$ $\left(\mathrm{MNa}^{+}, 100\right)$.

\section{ASSOCIATED CONTENT}

\section{S Supporting Information}

The Supporting Information is available free of charge on the ACS Publications website at DOI: 10.1021/acs.joc.6b01357.
${ }^{1} \mathrm{H}$ and ${ }^{13} \mathrm{C}$ NMR spectra for all novel compounds (PDF)

\section{AUTHOR INFORMATION}

\section{Corresponding Author}

*E-mail: Andrew.Sutherland@glasgow.ac.uk.

\section{Notes}

The authors declare no competing financial interest.

\section{ACKNOWLEDGMENTS}

Financial support from the Ministry of Higher Education and Scientific Research and the University of Benghazi, Libya (studentship to S.A.I.S.), the EPSRC (studentship to E.D.D.C., EP/P505534/1), the Coordenação de Aperfeiçoamento de Pessoal de Nivel Superior (CAPES, grant to F.G.D., Proc. No. $88888.021508 / 2013-00$ ), and Science without Borders is gratefully acknowledged.

\section{REFERENCES}

(1) (a) Ryan, J. H.; Hyland, C.; Meyer, A. G.; Smith, J. A.; Yin, J.-X. Prog. Heterocycl. Chem. 2012, 24, 493. (b) Ramig, K. Tetrahedron 2013, 69, 10783.

(2) For examples of biologically active amino-substituted 1benzazepines, see: (a) Schoen, W. R.; Pisano, J. M.; Prendergast, K.; Wyvratt, M. J., Jr.; Fisher, M. H.; Cheng, K.; Chan, W. W.-S.; Butler, B.; Smith, R. G.; Ball, R. G. J. Med. Chem. 1994, 37, 897. (b) DeVita, R J.; Bochis, R.; Frontier, A. J.; Kotliar, A.; Fisher, M. H.; Schoen, W. R.; Wyvratt, M. J.; Cheng, K.; Chan, W. W.-S.; Butler, B.; Jacks, T. M.; Hickey, G. J.; Schleim, K. D.; Leung, K.; Chen, Z.; Chiu, S.-H. L.; Feeney, W. P.; Cunningham, P. K.; Smith, R. G. J. Med. Chem. 1998, 41, 1716. (c) Kondo, K.; Ogawa, H.; Yamashita, H.; Miyamoto, H.; Tanaka, M.; Nakaya, K.; Kitano, K.; Yamamura, Y.; Nakamura, S.; Onogawa, T.; Mori, T.; Tominaga, M. Bioorg. Med. Chem. 1999, 7, 1743. (d) Schultz, C.; Link, A.; Leost, M.; Zaharevitz, D. W.; Gussio, R.; Sausville, E. A.; Meijer, L.; Kunick, C. J. Med. Chem. 1999, 42, 2909. (e) Soto, S.; Vaz, E.; Dell'Aversana, C.; Álvarez, R.; Altucci, L.; de Lera, Á. R. Org. Biomol. Chem. 2012, 10, 2101.

(3) (a) Yamamura, Y.; Ogawa, H.; Yamashita, H.; Chihara, T.; Miyamoto, H.; Nakamura, S.; Onogawa, T.; Yamashita, T.; Hosokawa, T.; Mori, T.; Tominaga, M.; Yabuuchi, Y. Br. J. Pharmacol. 1992, 105, 787. (b) Ogawa, H.; Yamashita, H.; Kondo, K.; Yamamura, Y.; Miyamoto, H.; Kan, K.; Kitano, K.; Tanaka, M.; Nakaya, K.; Nakamura, S.; Mori, T.; Tominaga, M.; Yabuuchi, Y. J. Med. Chem. 1996, 39, 3547. (c) Matsubara, J.; Kitano, K.; Otsubo, K.; Kawano, Y.; Ohtani, T.; Bando, M.; Kido, M.; Uchida, M.; Tabusa, F. Tetrahedron 2000, 56, 4667. (d) Loison, S.; Cottet, M.; Orcel, H.; Adihou, H.; Rahmeh, R.; Lamarque, L.; Trinquet, E.; Kellenberger, E.; Hibert, M.; Durroux, T.; Mouillac, B.; Bonnet, D. J. Med. Chem. 2012, 55, 8588.

(4) Cao, G.; Escribano, A. M.; Fernandez, M. C.; Fields, T.; Genert, D. L.; Cioff, C. L.; Herr, R. J.; Mantlo, N. B.; Martin, D. L. N. E. M.; Mateo, H. A. I.; Mayhugh, D. R.; Wang, X. Compounds and methods for treating dyslipidemia. W.O. Patent WO2005037796 A1, April 28, 2005.

(5) (a) Tabata, H.; Nakagomi, J.; Morizono, D.; Oshitari, T.; Takahashi, H.; Natsugari, H. Angew. Chem., Int. Ed. 2011, 50, 3075. (b) Tabata, H.; Yoneda, T.; Tasaka, T.; Ito, S.; Oshitari, T.; Takahashi, H.; Natsugari, H. J. Org. Chem. 2016, 81, 3136.

(6) For example, see: (a) Ohtani, T.; Kawano, Y.; Kitano, K.; Matsubara, J.; Komatsu, M.; Uchida, M.; Tabusa, F.; Nagao, Y. Heterocycles 2005, 66, 481. (b) Cordero-Vargas, A.; Quiclet-Sire, B.; Zard, S. Z. Bioorg. Med. Chem. 2006, 14, 6165. (c) Boeglin, D.; Bonnet, D.; Hibert, M. J. Comb. Chem. 2007, 9, 487. (d) Chang, M.-Y.; Chan, C.-K.; Lin, S.-Y. Heterocycles 2013, 87, 1519 and references therein. (e) Zhang, Y.; Zheng, L.; Yang, F.; Zhang, Z.; Dang, Q.; Bai, X. Tetrahedron 2015, 71, 1930. 
(7) (a) Kotha, S.; Shah, V. R. Eur. J. Org. Chem. 2008, 2008, 1054. (b) Sunderhaus, J. D.; Dockendorff, C.; Martin, S. F. Tetrahedron 2009, 65, 6454.

(8) (a) Calder, E. D. D.; McGonagle, F. I.; Harkiss, A. H.; McGonagle, G. A.; Sutherland, A. J. Org. Chem. 2014, 79, 7633.

(b) Calder, E. D. D.; Sharif, S. A. I.; McGonagle, F. I.; Sutherland, A. J. Org. Chem. 2015, 80, 4683.

(9) (a) Heck, R. F. Org. React. 1982, 27, 345. (b) Beletskaya, I. P.; Cheprakov, A. V. Chem. Rev. 2000, 100, 3009.

(10) Adepu, R.; Rajitha, A.; Ahuja, D.; Sharma, A. K.; Ramudu, B.; Kapavarapu, R.; Parsa, K. V. L; Pal, M. Org. Biomol. Chem. 2014, 12, 2514.

(11) (a) Caron, S.; Vazquez, E. J. Org. Chem. 2003, 68, 4104. (b) Yang, W.; He, H.-X.; Gao, Y.; Du, D.-M. Adv. Synth. Catal. 2013, 355,3670 .

(12) William, A. D.; Lee, A. C.-H.; Blanchard, S.; Poulsen, A.; Teo, E. L.; Nagaraj, H.; Tan, E.; Chen, D.; Williams, M.; Sun, E. T.; Goh, K. C.; Ong, W. C.; Goh, S. K.; Hart, S.; Jayaraman, R.; Pasha, M. K.; Ethirajulu, K.; Wood, J. M.; Dymock, B. W. J. Med. Chem. 2011, 54, 4638.

(13) Blanchette, M. A.; Choy, W.; Davis, J. T.; Essenfeld, A. P.; Masumune, S.; Roush, W. R.; Sakai, T. Tetrahedron Lett. 1984, 25, 2183.

(14) (a) Grafton, M. W.; Farrugia, L. J.; Senn, H. M.; Sutherland, A. Chem. Commun. 2012, 48, 7994. (b) Ahmad, S.; Sutherland, A. Org. Biomol. Chem. 2012, 10, 8251. (c) Grafton, M. W.; Farrugia, L. J.; Sutherland, A. J. Org. Chem. 2013, 78, 7199. (d) Grafton, M. W.; Johnson, S. A.; Farrugia, L. J.; Sutherland, A. Tetrahedron 2014, 70, 7133.

(15) (a) Scholl, M.; Ding, S.; Lee, C. W.; Grubbs, R. H. Org. Lett. 1999, 1, 953. (b) Sanford, M. S.; Love, J. A.; Grubbs, R. H. J. Am. Chem. Soc. 2001, 123, 6543.

(16) Saunthwal, R. K.; Patel, M.; Tiwari, R. K.; Parang, K.; Verma, A. K. Green Chem. 2015, 17, 1434.

(17) Hayashi, S.; Ueno, N.; Murase, A.; Nakagawa, Y.; Takada, J. Eur. J. Med. Chem. 2012, 50, 179.

(18) Youn, S. W.; Lee, S. R. Org. Biomol. Chem. 2015, 13, 4652.

(19) Sunke, R.; Kumar, V.; Ashfaq, M. A.; Yellanki, S.; Medisetti, R.; Kulkarni, P.; Ramarao, E. V. V. S.; Ehtesham, N. Z.; Pal, M. RSC Adv. 2015, 5, 44722. 